\title{
Separating the dynamical effects of climate change and ozone depletion. Part I: Southern Hemisphere stratosphere
}

Article

Published Version

McLandress, C., Jonsson, A. I., Plummer, D. A., Reader, M. C., Scinocca, J. F. and Shepherd, T. G. (2010) Separating the dynamical effects of climate change and ozone depletion. Part I: Southern Hemisphere stratosphere. Journal of Climate, 23 (18). pp. 5002-5020. ISSN 1520-0442 doi:

https://doi.org/10.1175/2010JCLI3586.1 Available at https://centaur.reading.ac.uk/31608/

It is advisable to refer to the publisher's version if you intend to cite from the work. See Guidance on citing.

To link to this article DOI: http://dx.doi.org/10.1175/2010JCLI3586.1

Publisher: American Meteorological Society

All outputs in CentAUR are protected by Intellectual Property Rights law, including copyright law. Copyright and IPR is retained by the creators or other copyright holders. Terms and conditions for use of this material are defined in the End User Agreement. 


\section{CentAUR}

Central Archive at the University of Reading

Reading's research outputs online 


\title{
Separating the Dynamical Effects of Climate Change and Ozone Depletion. Part I: Southern Hemisphere Stratosphere
}

\author{
Charles MCLANDRESS AND ANDREAS I. JONSSON \\ Department of Physics, University of Toronto, Toronto, Ontario, Canada \\ DAVID A. PlumMer \\ Canadian Centre for Climate Modelling and Analysis, Victoria, British Columbia, Canada \\ M. CATHERINE READER \\ University of Victoria, Victoria, British Columbia, Canada \\ JOHN F. SCINOCCA \\ Canadian Centre for Climate Modelling and Analysis, Victoria, British Columbia, Canada \\ THEODORE G. SHEPHERD \\ Department of Physics, University of Toronto, Toronto, Ontario, Canada
}

(Manuscript received 18 December 2009, in final form 1 April 2010)

\begin{abstract}
A version of the Canadian Middle Atmosphere Model that is coupled to an ocean is used to investigate the separate effects of climate change and ozone depletion on the dynamics of the Southern Hemisphere (SH) stratosphere. This is achieved by performing three sets of simulations extending from 1960 to 2099: 1) greenhouse gases (GHGs) fixed at 1960 levels and ozone depleting substances (ODSs) varying in time, 2) ODSs fixed at 1960 levels and GHGs varying in time, and 3) both GHGs and ODSs varying in time. The response of various dynamical quantities to the GHG and ODS forcings is shown to be additive; that is, trends computed from the sum of the first two simulations are equal to trends from the third. Additivity is shown to hold for the zonal mean zonal wind and temperature, the mass flux into and out of the stratosphere, and the latitudinally averaged wave drag in SH spring and summer, as well as for final warming dates. Ozone depletion and recovery causes seasonal changes in lower-stratosphere mass flux, with reduced polar downwelling in the past followed by increased downwelling in the future in SH spring, and the reverse in SH summer. These seasonal changes are attributed to changes in wave drag caused by ozone-induced changes in the zonal mean zonal winds. Climate change, on the other hand, causes a steady decrease in wave drag during SH spring, which delays the breakdown of the vortex, resulting in increased wave drag in summer.
\end{abstract}

\section{Introduction}

The combined effects of greenhouse gas (GHG) induced climate change and ozone depletion have had a significant impact on the stratosphere on both a global and a regional scale. Over the past three decades the

Corresponding author address: Charles McLandress, Dept. of Physics, University of Toronto, 60 St. George St., Toronto ON M5S 1A7, Canada.

E-mail: charles@atmosp.physics.utoronto.ca stratosphere has undergone a global average cooling of $0.5-1.5 \mathrm{~K} \mathrm{decade}^{-1}$, with substantially stronger cooling occurring in the Antarctic lower stratosphere in spring and summer (Randel et al. 2009). In conjunction with the high-latitude cooling there has also been a delay in the breakdown of the Southern Hemisphere $(\mathrm{SH})$ polar vortex (Waugh et al. 1999). These trends are attributed to increasing concentrations of $\mathrm{CO}_{2}$ and decreasing concentrations of ozone from chemical ozone depletion (Shine et al. 2003; Langematz et al. 2003). 
Separating the effects of climate change and ozone depletion is essential not only from the point of view of attribution but also for gaining a more complete understanding of the physical processes involved. While in principle this might be done by applying regression models to observations, the observational record is still not long enough to clearly separate the two effects (Stolarski et al. 2010). Thus, at present the only way to separate them is by using simulations from chemistry climate models (CCMs).

Here, there are several potential modeling strategies. One is to include all forcings in a single scenario in which both GHGs and ozone depleting substances (ODSs) vary transiently. The long-term effects of climate change, for example, can then be isolated by comparing a period far enough in the future with a period far enough in the past that the effects of ozone depletion can be ignored (e.g., Shepherd 2008; McLandress and Shepherd 2009, henceforth MS09). In addition, the separate effects of GHG and ODS changes over particular periods can be inferred from these simulations using regression analysis (e.g., Jonsson et al. 2009; Stolarski et al. 2010). However, regression analysis is associated with uncertainties due to the inherent limitations of the method; moreover, the statistical nature of the approach may obscure cause and effect if the explanatory variables are the physical fields themselves [e.g., temperature or ozone; Shepherd and Jonsson (2008)]. A second modeling strategy is to compare simulations in which either GHGs or ODSs are held fixed in time with simulations in which both forcings are allowed to vary (e.g., Garcia and Randel 2008; Perlwitz et al. 2008; Waugh et al. 2009; Oman et al. 2009).

The ideal approach, and the one that will be taken here, is to use three sets of simulations: two in which the effects of climate change and ozone depletion (and recovery) are induced separately, and a third in which the two effects are combined. Using three sets of simulations permits the additivity (i.e., linearity) of the responses to the GHG and ODS forcings to be examined. An additive response has important implications. First, it permits a simpler understanding of the physical mechanisms involved. Second, it means that only one of the two sets of sensitivity experiments need be performed for subsequent studies, which has an obvious practical benefit. Additivity also means that a separate suite of GHG experiments using weaker or moderately larger GHG forcings can then be combined with the ODS experiments to represent their combined effects.

On the other hand, a nonadditive response means that the combined forcings act together nonlinearly, in which case attribution becomes more complicated and interpretation of the results more difficult. An example of a possible nonadditive response would be if the increasing concentrations of GHGs were to extend the size of the region of the SH polar lower stratosphere where temperatures drop below the threshold for polar stratospheric clouds (PSCs) to form, causing additional heterogeneous chemical ozone loss. The resulting changes in ozone would then alter the zonal mean temperature (through radiation) and zonal wind (through thermal wind balance), which could in turn affect wave propagation and the residual circulation. Since changes in both GHGs and ODSs are required for this to happen (i.e., ozone loss in the extended region would not occur if only one of the forcings were to vary), the response would be nonadditive.

In setting up the CCM simulations, it is important that the sea surface temperatures and sea ice distribution (SSTs for short) be consistent with the anthropogenic forcings. What has generally been done is to use SSTs generated from coupled atmosphere-ocean models using the same GHG scenario as the CCMs. However, since the coupled models do not resolve the stratosphere, the impacts of the stratosphere on the SSTs are absent. Another common inconsistency is the use of observed SSTs for the past and modeled SSTs for the future (e.g., Li et al. 2008; Waugh et al. 2009), which may be problematic when comparing simulations of the past and the future because of discontinuous behavior at the transition point (which may not be identifiable in the face of interannual variability if the overlap period is restricted to only a decade or two) or changes in the nature of the atmospheric variability in the two periods. The ideal solution is to couple the CCM to an ocean. Not only does this ensure a seamless transition between past and future, it also allows for a two-way feedback between the ocean and atmosphere that is not present if the SSTs are prescribed. This feedback could have an important impact on the troposphere, especially in the $\mathrm{SH}$ where the impacts of the ozone hole are felt down to the surface (e.g., Gillett and Thompson 2003), which could in turn affect waves propagating up into the stratosphere.

In this two-part series of papers we analyze simulations from a version of the Canadian Middle Atmosphere Model (CMAM) that is coupled to an ocean. Three sets of simulations are examined: two in which either GHGs or ODSs are held fixed in time, and a third in which both vary transiently. The third set of simulations has been analyzed extensively as part of phase 2 of the Stratospheric Processes and their Role in Climate (SPARC) CCM Validation Activity (CCMVal-2; Eyring et al. 2008; SPARC CCMVal 2010). Since the dynamical effects of GHG-induced climate change have been examined in MS09 (using the previous version of CMAM), emphasis here is placed on the dynamical response to the ODS changes. The primary focus is on the $\mathrm{SH}$ where the effects of ozone depletion are largest. In this part of our two-part 
series, we consider the stratospheric response and examine zonal mean quantities like temperature, zonal wind, wave drag, and the residual circulation. Since the response to the ODS forcing is largely confined to the middle and high latitudes, we mainly focus on those regions, but we also show how changes in the wave drag in response to the ODS forcing affect the tropical upwelling branch of the Brewer-Dobson circulation. Throughout the paper we examine the additivity of the dynamical responses to the GHG and ODS forcings by comparing long-term trends from the sensitivity simulations to those from simulations using the combined forcings. In the forthcoming Part II (C. McLandress et al. 2010, unpublished manuscript), we extend our analysis to the troposphere, examining the impacts of the GHG and ODS forcings on features like the southern annular mode, jet location, tropopause height, and Hadley cell width.

\section{Description of model and simulations}

CMAM is the upward extension of the Canadian Centre for Climate Modelling and Analysis (CCCma) thirdgeneration coupled general circulation model (CGCM3). In addition to the interactive ocean and parameterizations inherent to the CCCma model, CMAM also includes an interactive stratospheric chemistry module and a comprehensive middle-atmosphere radiative transfer scheme. It has 71 levels in the vertical, with a resolution that varies from several hundred meters in the lower troposphere to $\sim 1.5 \mathrm{~km}$ near $20 \mathrm{~km}$, and to $\sim 2.5 \mathrm{~km}$ from $60 \mathrm{~km}$ to the model lid near $100 \mathrm{~km}$. In the horizontal, T31 spectral resolution is used, corresponding to a spacing of $\sim 6^{\circ}$ on the grid on which the physics and chemistry calculations are performed. Detailed descriptions of the chemistry scheme and the atmospheric component of CMAM are provided in de Grandpré et al. (2000) and Scinocca et al. (2008), respectively.

In contrast to previous versions of CMAM, this version is coupled to an ocean. The coupled model CGCM3 on which CMAM is based has been used extensively for the Intergovernmental Panel on Climate Change's (IPCC) Fourth Assessment Report. The ocean component of CGCM3 is based on a modified version of the National Center for Atmospheric Research's Community Ocean Model (NCOM1.3; Gent et al. 1998; Arora et al. 2009). It has a $1.86^{\circ}$ horizontal resolution, and 29 vertical levels whose thicknesses vary from $50 \mathrm{~m}$ in the upper layer to $300 \mathrm{~m}$ at the bottom. The coupled atmosphere-ocean version of CMAM has already been employed by Scinocca et al. (2009) and has been used extensively in SPARC CCMVal (2010). The latter includes a detailed analysis of CMAM, including its present-day climate and future projections. Of the 18 models discussed therein, CMAM was the only one coupled to an ocean; all others used prescribed SSTs.

The chemistry scheme includes all the relevant catalytic ozone loss cycles and is coupled, through ozone and $\mathrm{H}_{2} \mathrm{O}$, to the radiative transfer scheme. Following the standard specifications given in Eyring et al. (2008), the total amount of chlorine and bromine carried by longlived ODSs is distributed across the model ODSs. The ODS chemical tracers, in addition to other chemical tracers such as $\mathrm{CH}_{4}$ and $\mathrm{N}_{2} \mathrm{O}$, use a time-dependent mixing ratio as a lower boundary condition. Since this version of CMAM does not include tropospheric chemistry, chemical species below about $400 \mathrm{hPa}$ are treated as passive tracers. Heterogeneous reactions on stratospheric sulfate aerosols, liquid ternary solutions, and water ice are included, but there is no parameterization of nitric acid trihydrate (NAT) PSCs.

The reason why CMAM does not include a parameterization of NAT PSCs is twofold. First, there has never been a clear consensus on how to correctly represent NAT formation and sedimentation (see section 4.2.2 of WMO 2007). Indeed, Santee et al. (2008) have recently shown that the usual equilibrium models of NAT tend to overestimate the chlorine activation. Second, chlorine activates very effectively on liquid aerosols (supercooled ternary solution, STS) at low temperatures, which in contrast to NAT are well understood. In fact, Pitts et al. (2007) have shown that satellite observations over the Antarctic indicate a PSC area that is significantly smaller than what would be inferred from the commonly used temperature-based proxy $T_{\mathrm{NAT}}$, but which is similar in magnitude to that inferred from $T_{\mathrm{STS}}$. For these reasons, we do not expect the omission of a NAT parameterization in CMAM to lead to a systematic error in the representation of Antarctic ozone loss. Ozone loss in the Antarctic lower stratosphere in CMAM was found in fact to be well simulated (SPARC CCMVal 2010, chapter 6).

The radiative transfer scheme accounts for the effects of long-lived GHGs $\left(\mathrm{CO}_{2}, \mathrm{CH}_{4}, \mathrm{~N}_{2} \mathrm{O}, \mathrm{CFC}-11\right.$, and CFC-12), water vapor, and ozone. In the middle atmosphere the effects of non-local thermodynamic equilibrium are taken into account. In contrast to the chemistry scheme where three-dimensional fields of $\mathrm{CH}_{4}, \mathrm{~N}_{2} \mathrm{O}$, CFC-11, and CFC-12 are transported and prognosed, the radiation scheme uses prescribed globally averaged heightindependent values for these quantities. Similarly for $\mathrm{CO}_{2}$, time-dependent spatially uniform values are specified.

Three sets of simulations extending from 1950 to 2099 are discussed here, each comprising an ensemble of three members. All simulations were initialized in 1950 using a preindustrial spinup procedure for the ocean that employed the dynamical version of CMAM (i.e., prescribed ozone) following the method described by Scinocca et al. 
(2009). The detailed description of the initialization for the simulations discussed here can be found in Plummer et al. (2010) and is identical to that used for the CMAM contribution to SPARC CCMVal (2010). In 1950 the interactive chemistry is initialized and is used for the remainder of the integration. The first decade after the chemistry initialization (1950-60) is required to spin up the chemical tracers and so is not included in the analysis. The external forcings for each of the simulations discussed here follow the CCMVal-2 specifications given in Eyring et al. (2008).

The first set is the REF-B2 simulation. It employs timevarying concentrations of GHGs and ODSs, with the GHGs following the moderate IPCC Special Report on Emissions Scenarios (SRES) A1B scenario (Houghton et al. 2001) and the ODSs following the A1 scenario (WMO 2007). This set of simulations has been extensively analyzed in SPARC CCMVal (2010).

The second set is the GHG simulation (also referred to as $\mathrm{SCN}-\mathrm{B} 2 \mathrm{~b}$ ) in which the GHGs seen by the radiation code are allowed to vary in time as in the REF-B2 simulation, but ODSs are held fixed at their 1960 values in the chemistry code. The concentrations of CFC-11 and $\mathrm{CFC}-12$ passed to the radiation code evolve in the same manner as in REF-B2. This is done to maintain an identical radiative forcing to the REF-B2 simulation. This is a climate-change-only experiment since the low concentrations of the ODSs prevent any significant changes in halogen-driven ozone loss.

The third set is the ODS simulation (also referred to as SCN-B2c) in which the ODSs seen by the chemistry code are allowed to vary in time as in REF-B2, but the GHG concentrations, including the CFCs, used in the radiation code are held fixed at their 1960 levels. This is a (halogen driven) ozone-change-only experiment since the radiative impacts of all greenhouse gases is unchanging in time. To investigate the magnitude of any "committed" warming after 1960, we examined the global average temperature in the lower troposphere over the period 1960-79. This analysis revealed no detectable trend, indicating a stable climate for the coupled system.

In accordance with the CCMVal-2 specifications (Eyring et al. 2008) all three sets of simulations use prescribed background values for stratospheric sulfate aerosols; other external forcings such as eruptive volcanoes and solar cycle effects are not included. One drawback of the present set of ODS and GHG simulations is that they both include the chemical effects of transiently varying $\mathrm{N}_{2} \mathrm{O}$ and $\mathrm{CH}_{4}$. Consequently, an investigation of additivity using these runs will be subject to a "double counting" of the chemical effects of $\mathrm{N}_{2} \mathrm{O}$ and $\mathrm{CH}_{4}$ forcing. The impacts of double counting, however, are found to be small in the

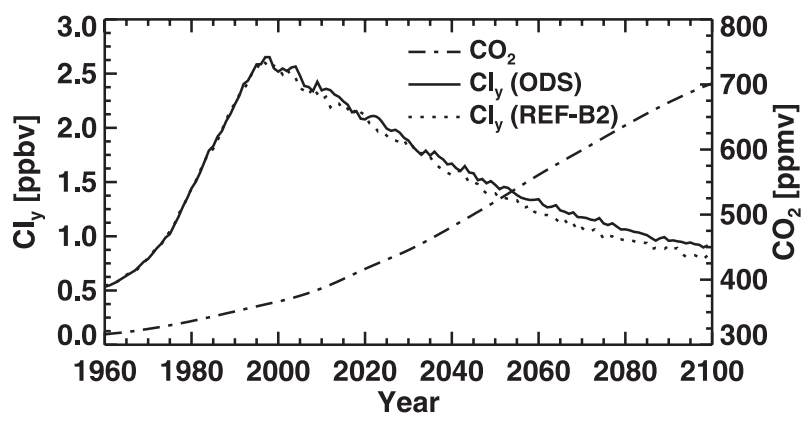

FIG. 1. Time series of total inorganic chlorine $\mathrm{Cl}_{\mathrm{y}}$ for the ensemble average of the ODS (solid) and REF-B2 (dotted) simulations at $50 \mathrm{hPa}$ averaged from $60^{\circ}$ to $90^{\circ} \mathrm{S}$ for SH spring (SON) and global and annual average $\mathrm{CO}_{2}$ (dashed-dotted). The $\mathrm{CO}_{2}$ is the prescribed forcing used in the GHG and REF-B2 simulations.

lower stratosphere, as will be shown in section 3a. There is also a weak double counting in the troposphere from timevarying tropospheric aerosols, the effects of which are implemented as a prescribed enhancement of the surface albedo in all three simulations.

To help assess the impacts of coupling CMAM to an ocean, we compare the results from the REF-B2 simulation to the REF-B1 simulation (Eyring et al. 2008). REF-B1 is a reference simulation of the recent past used to validate the CCMVal-2 models and is also extensively analyzed in SPARC CCMVal (2010). In addition to the GHG and ODS forcing used in REF-B2, the REF-B1 simulation uses observed SSTs and natural forcings of stratospheric sulfate aerosols related to explosive volcanoes and solar variability. The fact that REF-B1 and REF-B2 differ by more than just the SSTs makes it difficult to rigorously assess the impacts of the interactive ocean. Nevertheless, the comparison of the two simulations does yield some useful information, which is presented in section $3 b$.

In section 3 we will also make reference to MS09 with regard to the climate-change-induced changes in the resolved wave drag and the residual circulation. Although the results in MS09 were based on a different set of CMAM simulations, they are qualitatively similar to those discussed here, thus justifying our references to that study.

\section{Results}

Time series of the two primary forcing agents in these experiments, $\mathrm{Cl}_{\mathrm{y}}$ (total inorganic chlorine) and $\mathrm{CO}_{2}$, are shown in Fig. 1. For the ODS and REF-B2 simulations, $\mathrm{Cl}_{\mathrm{y}}$ is shown at $50 \mathrm{hPa}$ from $60^{\circ}$ to $90^{\circ} \mathrm{S}$ for SeptemberNovember (SON). Because it takes several years for the ODSs to be transported from the troposphere to the polar lower stratosphere, $\mathrm{Cl}_{\mathrm{y}}$ is a more appropriate 

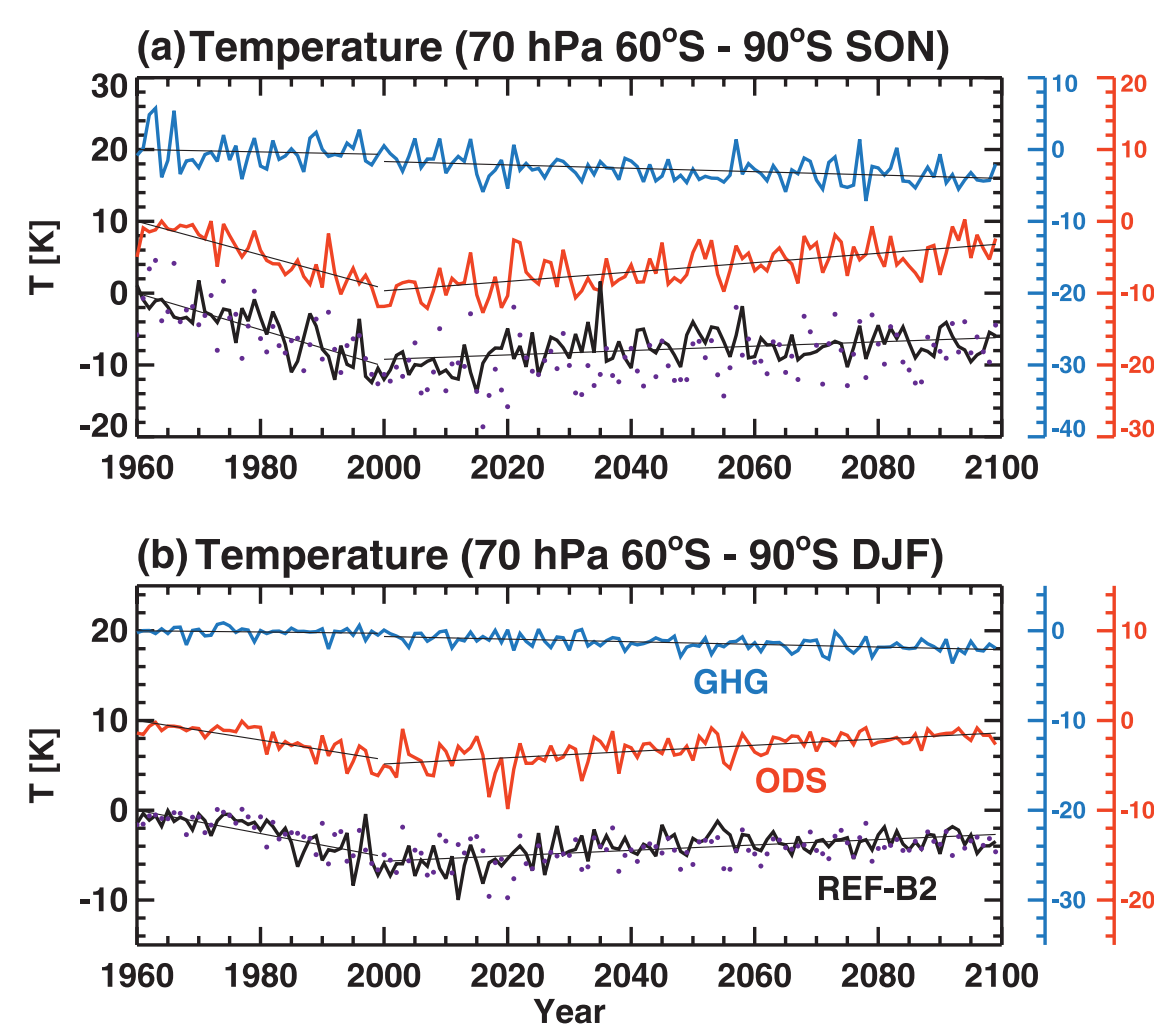

FIG. 2. Time series of polar cap average $\left(60^{\circ}-90^{\circ} \mathrm{S}\right)$ temperature anomalies at $70 \mathrm{hPa}$ for SH (a) spring (SON) and (b) summer (DJF): GHG (blue), ODS (red), and REF-B2 (black) simulations, and the sum of the GHG and ODS simulations (purple dots). For clarity the red and blue curves are shifted with respect to the black; their color-coded axes are given on the right. The straight lines are linear fits computed from 1960-99 and 2000-99. Ensemble averages are shown. Anomalies are computed with respect to the 1960 baselines given by the intercepts of the linear fits computed from 1960-99 (see text). The actual temperature time series can be recovered by adding the anomaly time series to the 1960 baselines, which for the REF-B2, GHG, and ODS simulations are, respectively, 206.9, 204.8, and $206.8 \mathrm{~K}$ for SON and 232.3, 231.2, and 232.2 for DJF.

indicator of the timing of halogen-driven ozone loss than is the ODS forcing at the surface. The $\mathrm{CO}_{2}$ concentration increases steadily throughout the simulation, whereas $\mathrm{Cl}_{\mathrm{y}}$ increases rapidly until the late 1990 s and decreases slowly thereafter. Note that in $2100, \mathrm{Cl}_{\mathrm{y}}$ is still larger than in 1960, a feature that is present in almost all CCMVal-2 models (SPARC CCMVal 2010, chapter 9) and that is consistent with the lower boundary condition for total organic chlorine used in the CCMVal-2 simulations. The more rapid decay with time of $\mathrm{Cl}_{\mathrm{y}}$ in the REF-B2 simulation is a consequence of climate change, which reduces the mean age of air in the stratosphere, leading to a smaller ratio of inorganic to total (inorganic plus organic) chlorine in the lower stratosphere. This effect, however, is small at the polar latitudes in the lower stratosphere shown here. The fact that $\mathrm{Cl}_{\mathrm{y}}$ peaks in the late 1990s motivates our choice of time periods used in the linear trend analysis, namely 1960-99 and 2000-99.

\section{a. Temperature and zonal wind}

Figure 2 shows time series of polar cap average $\left(60^{\circ}-\right.$ $90^{\circ} \mathrm{S}$ ) temperature at $70 \mathrm{hPa}$ for $\mathrm{SH}$ spring $(\mathrm{SON})$ and summer (December-February; DJF) as anomalies from their 1960 baseline values. These two seasons correspond to periods when the impacts of the ozone hole are expected to be largest. For clarity the results from the two sensitivity experiments (colored curves) are shifted with respect to REF-B2 (black curves); their respective axes are plotted on the right-hand side of Fig. 2. The baseline values used to create the anomaly time series are defined as the 1960 intercepts of the linear fits computed from the ensemble average time series from 1960 to 1999. Differences between the baseline values for the three ensembles are small and are related to statistical uncertainty in the intercept values.

Ozone depletion and recovery only (red curves in Fig. 2) result in a rapid cooling in the twentieth century, followed 
TABLE 1. Linear trends in zonal mean temperature $(\bar{T})$ at $70 \mathrm{hPa}$, residual vertical velocity $\left(\bar{w}^{*}\right)$ at $70 \mathrm{hPa}$, and Eliassen-Palm flux divergence $(\bar{D})$ at $50 \mathrm{hPa}$. Area averages from $60^{\circ}$ to $90^{\circ} \mathrm{S}$ are used for $\bar{T}$ and $\bar{w}^{*}$, and from $40^{\circ}$ to $80^{\circ} \mathrm{S}$ for $\bar{D}$. The second to last column is for the sum of the GHG and ODS simulations. Uncertainties correspond to the $95 \%$ confidence levels. Units are $\mathrm{K}^{-1 e c a d e}{ }^{-1}$, $10^{-2} \mathrm{~mm} \mathrm{decade}^{-1}$, and $10^{-2} \mathrm{~m} \mathrm{~s}^{-1}$ day $^{-1}$ decade $^{-1}$.

\begin{tabular}{|c|c|c|c|c|c|c|c|}
\hline & Season & Period & GHG & ODS & REF-B2 & GHG + ODS & REF-B1 \\
\hline \multirow[t]{3}{*}{$\bar{T}$} & SON & 1960-99 & $-0.2 \pm 0.6$ & $-2.3 \pm 0.5$ & $-2.5 \pm 0.6$ & $-2.5 \pm 0.8$ & $-2.5 \pm 0.8$ \\
\hline & & 2000-99 & $-0.2 \pm 0.1$ & $0.7 \pm 0.2$ & $0.3 \pm 0.1$ & $0.4 \pm 0.2$ & - \\
\hline & & 1960-2099 & $-0.3 \pm 0.1$ & - & - & - & - \\
\hline \multirow[t]{3}{*}{$\bar{T}$} & DJF & 1960-99 & $-0.1 \pm 0.2$ & $-1.1 \pm 0.3$ & $-1.3 \pm 0.4$ & $-1.2 \pm 0.3$ & $-1.2 \pm 0.3$ \\
\hline & & $2000-99$ & $-0.1 \pm 0.0$ & $0.3 \pm 0.1$ & $0.3 \pm 0.1$ & $0.2 \pm 0.1$ & - \\
\hline & & 1960-2099 & $-0.2 \pm 0.0$ & - & - & - & - \\
\hline \multirow[t]{3}{*}{$\bar{w}^{*}$} & SON & 1960-99 & $-0.2 \pm 1.6$ & $0.8 \pm 1.7$ & $1.4 \pm 1.5$ & $0.6 \pm 2.5$ & $0.9 \pm 1.5$ \\
\hline & & $2000-99$ & $0.0 \pm 0.3$ & $-0.4 \pm 0.4$ & $0.1 \pm 0.4$ & $-0.4 \pm 0.6$ & - \\
\hline & & 1960-2099 & $0.3 \pm 0.2$ & - & - & - & - \\
\hline \multirow[t]{3}{*}{$\bar{w}^{*}$} & DJF & 1960-99 & $-0.7 \pm 0.9$ & $-4.1 \pm 1.0$ & $-5.3 \pm 1.2$ & $-4.8 \pm 1.5$ & $-4.9 \pm 1.0$ \\
\hline & & 2000-99 & $-0.4 \pm 0.2$ & $1.3 \pm 0.3$ & $0.7 \pm 0.2$ & $0.9 \pm 0.3$ & - \\
\hline & & 1960-2099 & $-0.6 \pm 0.1$ & - & - & - & - \\
\hline \multirow[t]{3}{*}{$\bar{D}$} & SON & 1960-99 & $0.7 \pm 3.9$ & $4.7 \pm 3.8$ & $6.6 \pm 3.7$ & $5.4 \pm 6.0$ & $6.5 \pm 3.6$ \\
\hline & & $2000-99$ & $1.2 \pm 0.8$ & $-1.9 \pm 0.8$ & $0.2 \pm 0.8$ & $-0.7 \pm 1.2$ & - \\
\hline & & 1960-2099 & $1.6 \pm 0.5$ & - & - & - & - \\
\hline \multirow[t]{3}{*}{$\bar{D}$} & DJF & 1960-99 & $-1.8 \pm 2.9$ & $-8.6 \pm 2.7$ & $-12.3 \pm 3.0$ & $-10.4 \pm 4.6$ & $-10.1 \pm 2.4$ \\
\hline & & $2000-99$ & $-1.5 \pm 0.5$ & $2.4 \pm 0.6$ & $0.1 \pm 0.6$ & $0.9 \pm 0.8$ & - \\
\hline & & 1960-2099 & $-2.2 \pm 0.3$ & - & - & - & - \\
\hline
\end{tabular}

by a gradual warming to the end of the twenty-first century. The temperature changes are largest in SON when ozone changes (and consequently solar heating changes) are largest; similar behavior occurs in DJF but with weaker trends. Since the chlorine loading in the ODS simulation is larger in 2100 than in 1960 (Fig. 1), the temperature at the end of the ODS simulation is slighter lower than at the beginning due to weaker solar heating as a result of lower ozone concentrations. Climate change only (blue curves) produces a weak but monotonic decrease in temperature throughout the entire simulation, which is larger in SON than in DJF. The larger trend in SON results from the combined effects of increased $\mathrm{CO}_{2}$ cooling and reduced dynamical heating, as discussed in MS09.

Figure 2 also indicates that the time evolution of the temperature is fairly linear, suggesting that a piecewise linear trend model should provide a reasonable trend estimate. The straight lines shown in Fig. 2 are computed using least squares fits from 1960-99 and 2000-99. Table 1 (columns 4-6, rows 2-7) lists the polar cap temperature trends for SON and DJF for the three simulations, along with their uncertainties, which are computed from the 95\% confidence levels. The resulting trends are insensitive to the precise date chosen for the pivot year provided that it is near the peak of the $\mathrm{Cl}_{\mathrm{y}}$ time series.

To investigate the additivity of the time series, we sum the temperature anomaly time series for the ODS and GHG simulations. These sums, which are given by the dotted curves in Fig. 2, are seen to overlay the REF-B2 time series. Ignoring the year-to-year fluctuations, which are irrelevant since we are interested in the long-term behavior, the overall agreement between the two pairs of curves is good, suggesting that the temperature response to the GHG and ODS changes is additive at this location. This is quantified in the second to last column in Table 1, which lists the trends and uncertainties for the sum of the ODS and GHG time series computed over the two time periods. For both periods the trends in the sum are in close agreement with those for REF-B2, indicating that the temperature response at this location is indeed additive, within the statistical uncertainty.

The top and bottom panels, respectively, of Fig. 3 show the zonal mean temperature trends for $\mathrm{SH}$ spring (SON) versus latitude and pressure for the past (1960-99) and future (2000-99). Considering first the ODS simulation (Figs. 3b and 3f), the region of strong cooling in the past near $50 \mathrm{hPa}$ and $90^{\circ} \mathrm{S}$ results from heterogeneous chemical ozone depletion in the Antarctic ozone hole, while the broader region of weaker cooling at mid- to low latitudes in the upper stratosphere results from gas-phase chemical ozone depletion; the trends in these two regions are statistically significant, as indicated by the shading. In the future these trends reverse, albeit at a slower rate due to the more gradual decline of $\mathrm{Cl}_{\mathrm{y}}$. The corresponding results for the GHG simulation (Figs. 3a and 3e) exhibit warming in the troposphere and cooling throughout the stratosphere, which increases with height. These trends reflect the radiative response to increasing GHG concentrations. In the mid- to lower stratosphere in the GHG simulation, the cooling trends are larger at high latitudes than at low latitudes. This is a consequence of the 


\section{Zonal mean temperature trends (SON)}
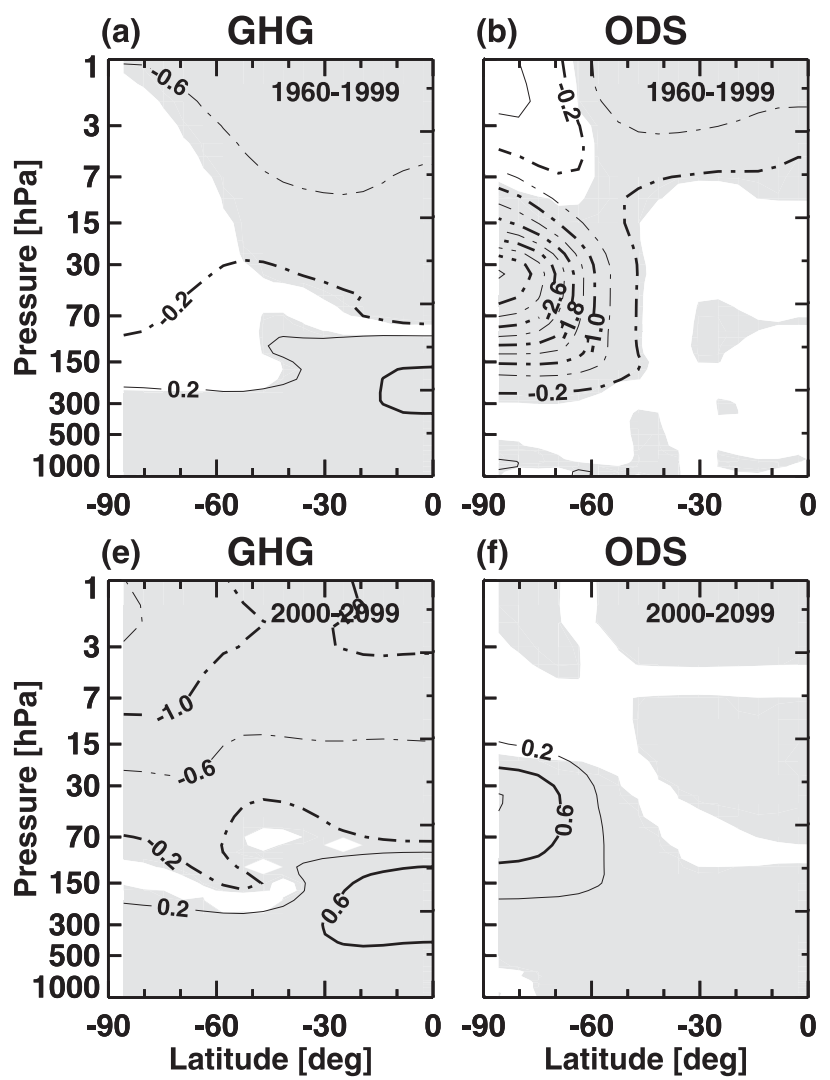

(f)

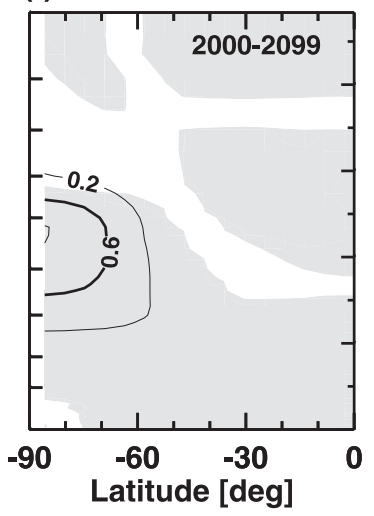

(c) REF-B2

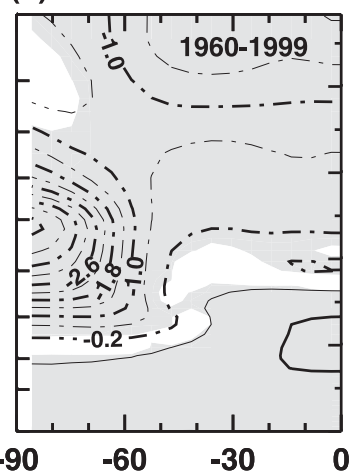

(g)

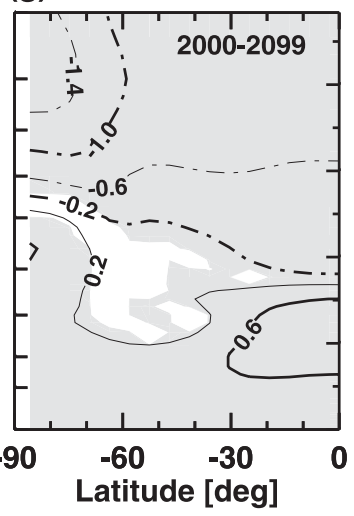

(d) $\mathrm{GHG}+$ ODS

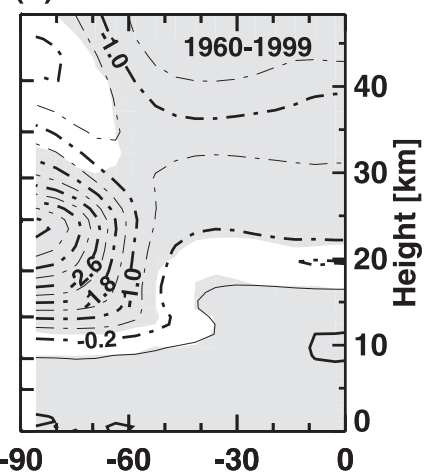

(h) $G H G$ + ODS

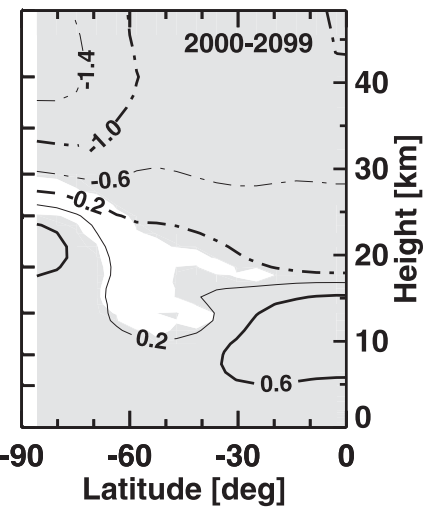

FIG. 3. Linear trends in zonal mean temperature vs latitude and pressure for SH spring (SON): (a),(e) GHG, (b),(f) ODS, and (c),(g) REF-B2 simulations; (d),(h) sum of the GHG and ODS simulations. Trends are computed from (top) 1960-99 and (bottom) 2000-99. Contour interval is $0.4 \mathrm{~K} \mathrm{decade}^{-1}$. Gray shading denotes confidence levels of $\geq 95 \%$ that the trends are different from zero. Log-pressure height, computed using a 7-km scale height, is plotted at the right. Ensemble averages are shown.

weakening dynamical heating brought about by weakening planetary wave drag that occurs in this simulation in response to climate change as discussed in MS09.

The far-right panels in Fig. 3 show the trends for the sum of the GHG and ODS simulations. The good agreement with the REF-B2 results (adjacent panels) indicates that the response is very nearly additive. This is seen more clearly in Fig. 4, which shows past and future trends computed from time series generated from the sum of the two sensitivity experiments minus REF-B2. To highlight the differences, a smaller contour interval than in Fig. 3 is used. In all regions the trends in Fig. 4 are much smaller (less than 20\%) than those of REF-B2, indicating that the stratospheric temperature response to climate change and ozone depletion and recovery is additive to leading order. Shading in Fig. 4 denotes regions where the trends are significantly different from zero, indicating that there is a (small) nonadditive response. In the past (Fig. 4a), the trends are not statistically significant, indicating that if there is a nonadditive response, it cannot be distinguished from the noise. In the future (Fig. 4b), however, the trends at low latitudes in the middle and upper stratosphere are statistically significant, indicating that the trends are nonadditive. This nonadditivity most likely arises because of double counting of the chemical effects of time-varying $\mathrm{N}_{2} \mathrm{O}$ and $\mathrm{CH}_{4}$ discussed in section 2, and not because of a nonlinear response to the forcings. The effect, however, is small. There is also a region of weak $(5 \%-10 \%)$ nonadditivity in the troposphere (outside of our region of interest). A likely explanation for this is the double-counting effect of the prescribed tropospheric aerosol forcing that was mentioned in section 2; since that forcing peaks in about 2020 , this would explain the weak net warming over this century seen in Fig. $4 b$.

Since changes in temperature imply changes in zonal wind, one would expect that additivity of the zonal wind response would also hold. This is indeed the case as seen in Fig. 5, which shows the zonal mean zonal wind trends for SON for the past and future: a comparison of the trends computed from REF-B2 to the sum of the two sensitivity experiments (third and fourth columns in Fig. 5) 
(a) 1960-1999

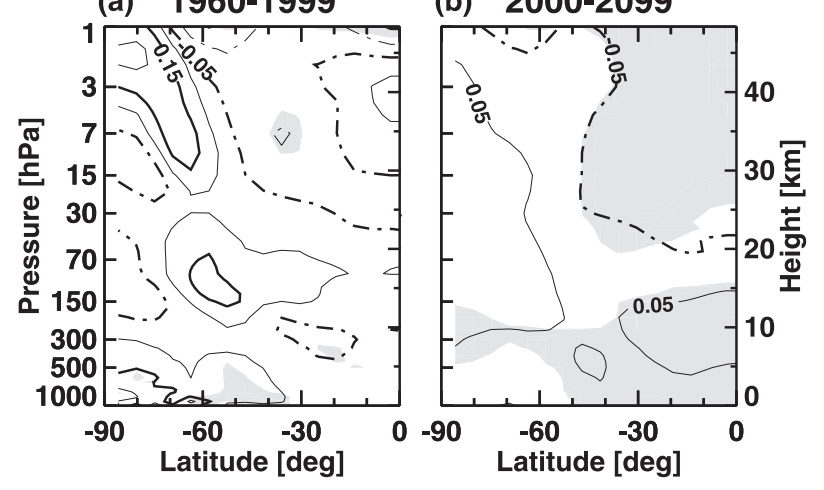

FIG. 4. Linear trends in zonal mean temperature vs latitude and pressure for SON for the sum of the GHG and ODS simulations minus REF-B2: (a) 1960-99 and (b) 2000-99. Contour interval is $0.1 \mathrm{~K} \mathrm{decade}^{-1}$. Gray shading denotes confidence levels $\geq 95 \%$ that the trends are different from zero. Ensemble averages are shown.

shows qualitatively good agreement. The past cooling due to ozone depletion accelerates the stratospheric westerlies at mid- to high latitudes (Fig. 5b). Climate change, on the other hand, results in a strengthening of the upper flank of the subtropical tropospheric jet (Fig. 5a), which extends high into the stratosphere in the future (Fig. 5e). While the trends in the ODS simulation change sign in the future, those in the GHG simulation remain the same.

The above conclusions about additivity in the $\mathrm{SH}$ stratosphere also hold for DJF (results not shown). In the next section we provide tabulated results at specific locations showing that additivity also holds for a number of other dynamical quantities.

\section{b. Brewer-Dobson circulation}

The transformed Eulerian mean residual velocity $\bar{v}^{*}$ and $\bar{w}^{*}$ (Andrews et al. 1987) is typically used as a proxy for the Brewer-Dobson circulation. The net mass flux entering the stratosphere just above the tropical tropopause that is computed from $\bar{w}^{*}$ provides a useful way of quantifying the overall strength of the circulation (Holton 1990), and has been used in a number of studies (e.g., Butchart et al. 2006; Li et al. 2008; MS09). The net downward mass flux in the two hemispheres is defined as

$$
\begin{aligned}
& F_{m}^{\mathrm{sh}}=-2 \pi a^{2} \rho \int_{-\pi / 2}^{\phi_{t}^{\mathrm{sh}}} \bar{w}^{*} \cos \phi d \phi, \quad \text { and } \\
& F_{m}^{\mathrm{nh}}=-2 \pi a^{2} \rho \int_{\phi_{t}^{n h}}^{\pi / 2} \bar{w}^{*} \cos \phi d \phi,
\end{aligned}
$$

where $a$ is the earth's radius, $\rho$ the background density in $\log$-pressure coordinates (Andrews et al. 1987), and $\phi_{t}^{\text {sh }}$ and $\phi_{t}^{\mathrm{nh}}$ are the so-called turnaround latitudes where tropical upwelling changes to extratropical downwelling.
The turnaround latitudes vary between $20^{\circ}$ and $30^{\circ}$ depending upon the season, extending farther poleward in the summer hemisphere. The net upward mass flux, which occurs in the tropics and subtropics, follows from the constraint of zero global average mass flux (on a constant pressure surface) and is given by $F_{m}^{\mathrm{tr}}=F_{m}^{\mathrm{nh}}+F_{m}^{\mathrm{sh}}$.

Figure 6 shows time series of the net upward mass flux at $70 \mathrm{hPa}$ for SON and DJF for the three sets of simulations. As in Fig. 2, anomalies with respect to a 1960 baseline are shown. The GHG simulation (blue curves) has a steadily increasing net upward mass flux that exhibits no significant change of slope between the past and future. That the slope is similar to that of REF-B2 (black curves) reconfirms that climate change is largely responsible for the acceleration of the Brewer-Dobson circulation. The ODS simulation (red curves) has a much different behavior. Not only do the trends change sign near 2000, but their seasonal dependence also changes: in SON a negative trend before 2000 is followed by a small but statistically significant (see Table 2) positive trend, while in DJF the reverse occurs.

A comparison of the net upward mass flux in the REFB2 simulation (black curve in Fig. 6) with the sum of the net upward mass fluxes in the ODS and GHG simulations (purple dots) suggests that additivity also holds for this quantity. This is quantified in Table 2, which lists the net upward mass flux trends and uncertainties for all seasons, as well as the annual mean: within the statistical uncertainty, additivity holds. In March-May (MAM) and June-August (JJA) the trends for the ODS simulation are substantially weaker than those for the GHG simulation, indicating that climate change is by far the largest driver of the trends in those seasons. In SON and DJF, the trends for the two sensitivity experiments are more similar in magnitude, but with those for the ODS simulation being of opposite sign in the two seasons. Because of this sign change, partial cancelation occurs when the annual mean is computed, resulting in annual mean trends that are driven by climate change.

Table 3 lists the SH net downward mass flux trends for the different simulations. Comparison to Table 2 indicates that virtually all of the trend in upward mass flux in the ODS simulation is due to the SH trends. In the REF-B2 simulation the much larger downward mass flux trend in DJF in the past explains the similar behavior seen in the upward mass flux.

To see how much of the SH net downward mass flux is occurring over the polar cap, Fig. 7 shows time series of the net downward mass flux versus the downward mass flux computed from $60^{\circ}$ to $90^{\circ} \mathrm{S}$. To aid the eye, the polar cap mass flux curve has been shifted by a constant amount (see the caption for Fig. 7). In the case of the ODS simulation (Fig. 7, left panels) the two time series overlay 


\section{Zonal mean zonal wind trends (SON)}

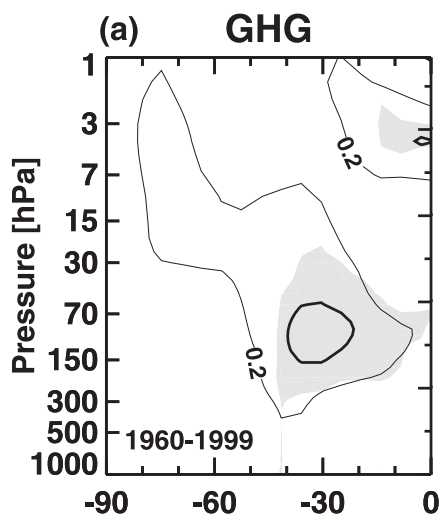

(b) ODS
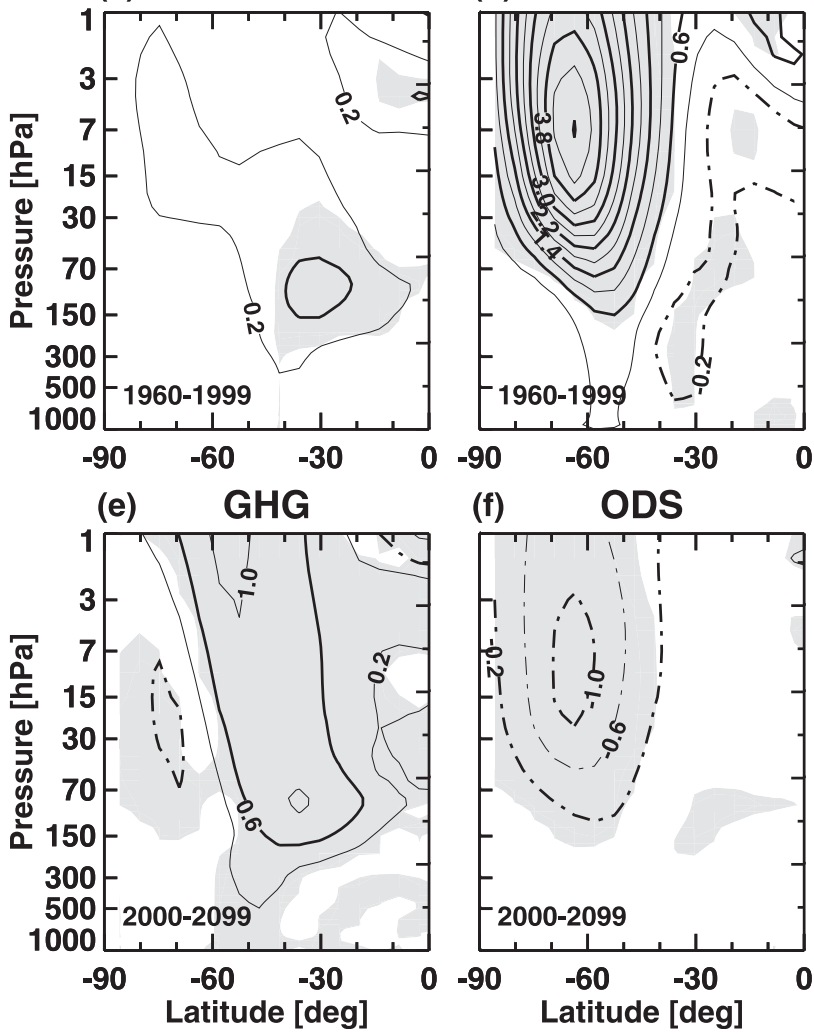

(f)

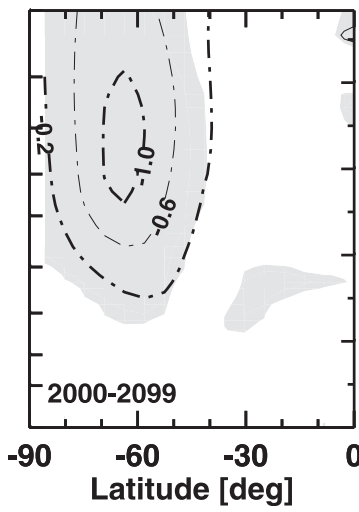

(c) REF-B2

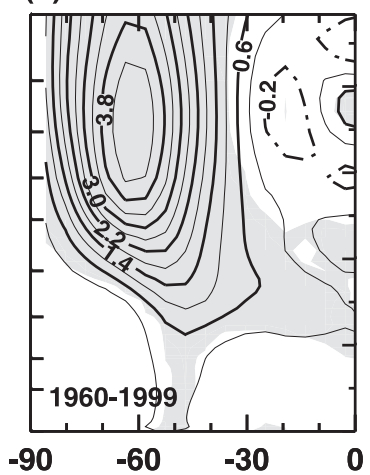

(g) REF-B2

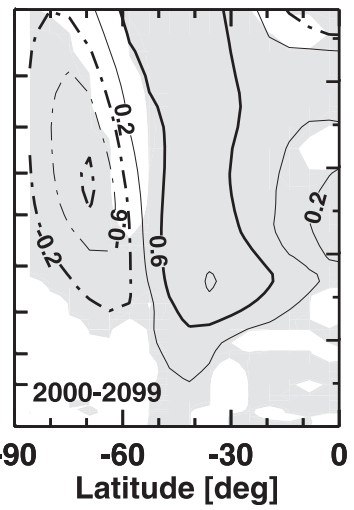

(d) $\mathrm{GHG}+$ ODS

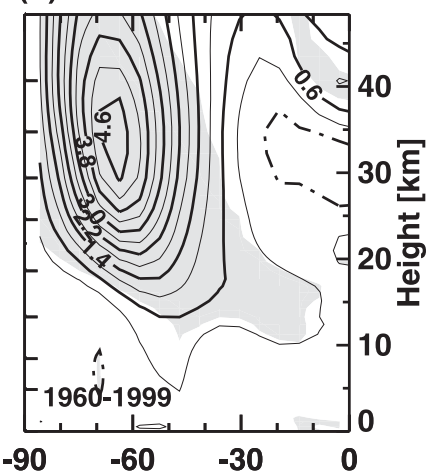

(h) $G H G+O D S$

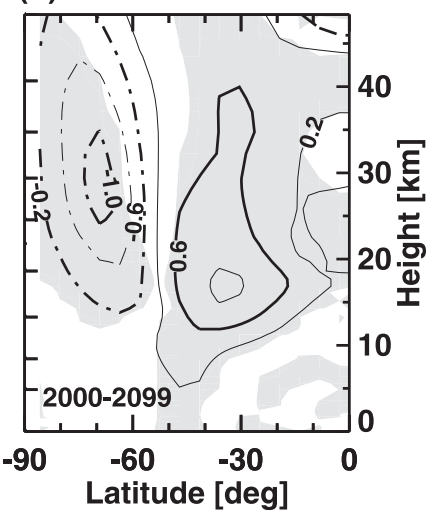

FIG. 5. As in Fig. 3, but for zonal mean zonal wind. Contour interval is $0.4 \mathrm{~m} \mathrm{~s}^{-1}$ decade $^{-1}$.

each other almost perfectly, indicating that virtually all of the temporal variations in the SH net downward mass flux are occurring over the polar cap. Thus, for the ODS simulation, both the short- and long-term changes in the net upward mass flux seen in Fig. 6 are due almost entirely to downwelling over the polar cap.

In the case of the GHG simulation (Fig. 7, right panels) the agreement between the two curves is not as good, indicating that changes in downwelling equatorward of $60^{\circ} \mathrm{S}$ are occurring. This is particularly evident in DJF, which shows a much weaker trend for the polar cap average as compared to the net value. This indicates that the dominant effect of GHG-induced climate change is to strengthen the downwelling at the subtropical latitudes and midlatitudes, which is consistent with the findings of MS09.

One of the impacts of coupling CMAM to an ocean model is a more rapid warming of the troposphere than in the uncoupled version: the global average temperature trend from 1960 to 2004 is about twice as large in REF-B2 as it is in REF-B1, which is consistent with the differences in the corresponding SST trends. The reasons for this are not fully understood and are currently under investigation. As a result, there is a marked difference in the lower-stratospheric net upward mass flux trends between REF-B2 and REF-B1, as seen in Table 2. The annual mean trend is about $40 \%$ larger in REF-B2 than in REF-B1. However, an examination of the trends in net downward mass flux in the $\mathrm{SH}$ listed in Table 3 shows much closer agreement between REF-B1 and REF-B2, indicating that the differences seen in the net upward mass flux are primarily attributed to $\mathrm{NH}$ changes. The linear trends in other physical quantities analyzed in this study are also listed for the REF-B1 simulation in Table 1. A comparison to the REF-B2 results for the past shows almost no difference in the trends (within statistical uncertainty). This indicates that the past effects of climate change on mid- to high latitudes in the $\mathrm{SH}$ are not significantly different for the coupled and uncoupled versions of the model.

In the next two subsections we examine the causes for the SH mass flux changes, focusing on changes in wave drag and mean winds at middle and high latitudes. We start by discussing the impacts of ozone depletion and recovery, using the ODS simulation, and then move on to the impacts of climate change, using the GHG simulation. 
(a) Net upward mass flux (70 hPa SON)

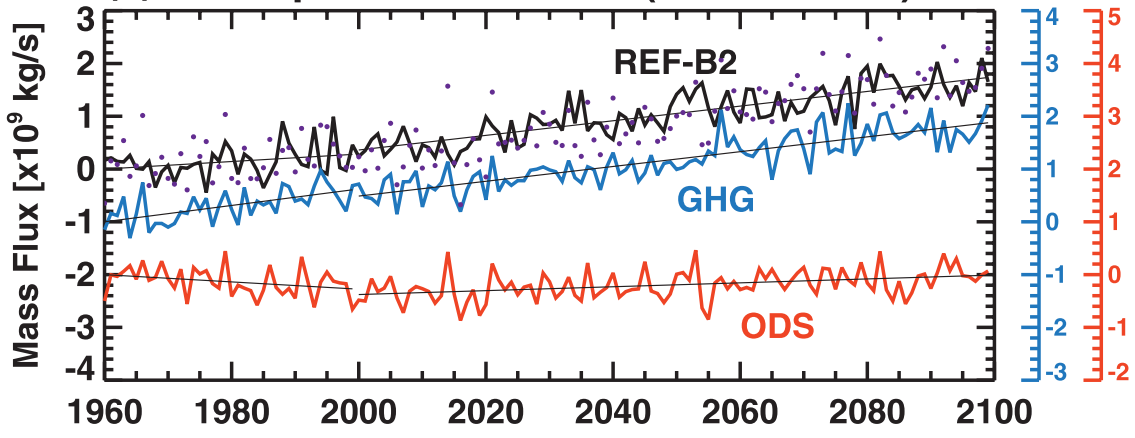

(b) Net upward mass flux (70 hPa DJF)

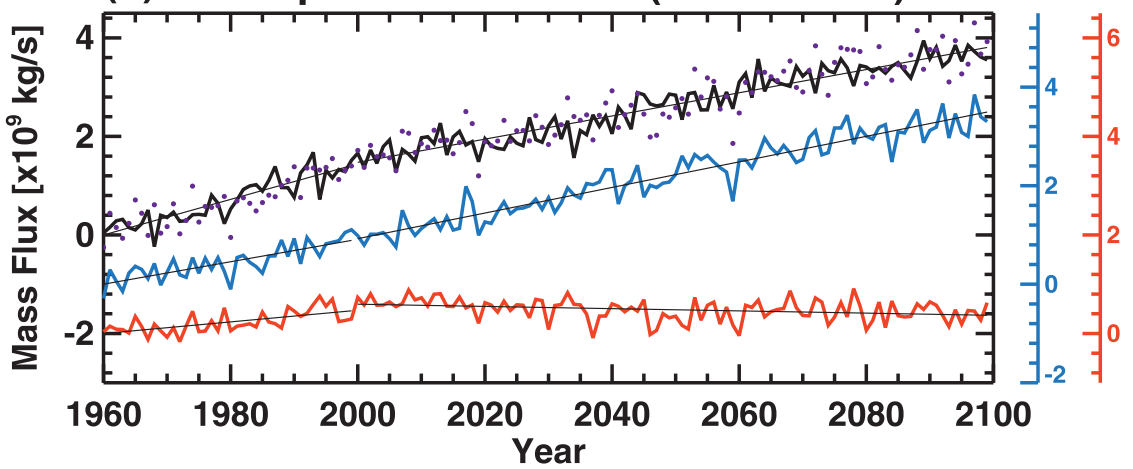

FIG. 6. Time series of net upward mass flux anomalies at $70 \mathrm{hPa}$ for (a) SON and (b) DJF: GHG (blue), ODS (red), and REF-B2 (black) simulations, and the sum of the GHG and ODS simulations (purple dots). Anomalies are computed with respect to 1960 baselines, which for the REF-B2, GHG, and ODS simulations are, respectively, 7.70, 7.58, and $7.80 \times 10^{9} \mathrm{~kg} \mathrm{~s}^{-1}$ for SON and 8.18, 8.40, and $8.36 \times 10^{9} \mathrm{~kg} \mathrm{~s}^{-1}$ for DJF. See the Fig. 2 caption for more details.

1) IMPACTS OF OZONE DEPLETION AND RECOVERY

Motivated by the above findings that the changes in tropical upwelling in the ODS simulation are a consequence of changes in downwelling at high latitudes in the SH, Figs. 8a and 9a show time series of $\bar{w}^{*}$ anomalies at $70 \mathrm{hPa}$ over the polar cap for the ODS simulation (red curves). Changes in the downwelling are brought about by changes in the resolved wave drag in

TABLE 2. Linear trends in net upward mass flux at $70 \mathrm{hPa}$. The second to last column is for the sum of the GHG and ODS simulations. Uncertainties correspond to the $95 \%$ confidence levels. Units are $10^{9} \mathrm{~kg} \mathrm{~s}^{-1} \mathrm{yr}^{-1}$.

\begin{tabular}{|c|c|c|c|c|c|c|}
\hline Season & Period & GHG & ODS & REF-B2 & $\mathrm{GHG}+\mathrm{ODS}$ & REF-B1 \\
\hline \multirow[t]{3}{*}{ SON } & 1960-99 & $15.5 \pm 7.0$ & $-6.9 \pm 7.1$ & $7.1 \pm 8.2$ & $8.6 \pm 10.8$ & $5.8 \pm 8.1$ \\
\hline & 2000-99 & $14.0 \pm 1.8$ & $3.7 \pm 1.7$ & $13.9 \pm 1.9$ & $17.7 \pm 2.7$ & - \\
\hline & 1960-2099 & $13.2 \pm 1.1$ & - & - & - & - \\
\hline \multirow[t]{3}{*}{ DJF } & 1960-99 & $22.8 \pm 5.6$ & $11.8 \pm 4.8$ & $36.1 \pm 6.1$ & $34.6 \pm 7.5$ & $28.9 \pm 9.4$ \\
\hline & 2000-99 & $26.0 \pm 1.7$ & $-2.3 \pm 1.4$ & $23.6 \pm 1.6$ & $23.7 \pm 2.2$ & - \\
\hline & 1960-2099 & $25.4 \pm 1.0$ & - & - & - & - \\
\hline \multirow[t]{3}{*}{ MAM } & 1960-99 & $17.8 \pm 3.2$ & $0.8 \pm 3.6$ & $21.6 \pm 2.5$ & $18.7 \pm 5.0$ & $11.6 \pm 4.8$ \\
\hline & 2000-99 & $20.9 \pm 1.0$ & $1.6 \pm 0.8$ & $20.4 \pm 1.1$ & $22.4 \pm 1.4$ & - \\
\hline & 1960-2099 & $20.0 \pm 0.6$ & - & - & - & - \\
\hline \multirow[t]{3}{*}{ JJA } & 1960-99 & $13.5 \pm 4.8$ & $1.6 \pm 4.7$ & $12.8 \pm 4.2$ & $15.1 \pm 6.7$ & $5.5 \pm 5.2$ \\
\hline & 2000-99 & $17.2 \pm 1.1$ & $1.8 \pm 1.1$ & $16.5 \pm 1.2$ & $19.0 \pm 1.5$ & - \\
\hline & 1960-2099 & $16.1 \pm 0.7$ & - & - & - & - \\
\hline \multirow[t]{3}{*}{ Annual } & 1960-99 & $17.4 \pm 2.7$ & $1.8 \pm 2.4$ & $19.4 \pm 3.4$ & $19.2 \pm 4.0$ & $12.9 \pm 4.5$ \\
\hline & 2000-99 & $19.5 \pm 0.8$ & $1.2 \pm 0.7$ & $18.6 \pm 0.8$ & $20.7 \pm 1.1$ & - \\
\hline & 1960-2099 & $18.7 \pm 0.5$ & - & - & - & - \\
\hline
\end{tabular}


TABLE 3. Linear trends in SH net downward mass flux at $70 \mathrm{hPa}$. The second to last column is for the sum of the GHG and ODS simulations. Uncertainties correspond to the $95 \%$ confidence levels. Units are $10^{9} \mathrm{~kg} \mathrm{~s}^{-1} \mathrm{yr}^{-1}$. Negative trends indicate decreasing downwelling.

\begin{tabular}{|c|c|c|c|c|c|c|}
\hline Season & Period & GHG & ODS & REF-B2 & $\mathrm{GHG}+\mathrm{ODS}$ & REF-B1 \\
\hline \multirow[t]{3}{*}{ SON } & 1960-99 & $5.7 \pm 7.9$ & $-7.0 \pm 7.1$ & $-3.3 \pm 8.4$ & $-1.3 \pm 11.6$ & $-1.5 \pm 7.5$ \\
\hline & 2000-99 & $1.7 \pm 1.8$ & $3.1 \pm 1.9$ & $2.2 \pm 2.0$ & $4.9 \pm 2.9$ & - \\
\hline & 1960-2099 & $1.3 \pm 1.1$ & - & - & - & - \\
\hline \multirow[t]{3}{*}{ DJF } & 1960-99 & $5.4 \pm 3.2$ & $11.6 \pm 3.6$ & $21.3 \pm 4.0$ & $17.1 \pm 5.4$ & $17.0 \pm 4.7$ \\
\hline & 2000-99 & $6.8 \pm 0.6$ & $-3.6 \pm 0.9$ & $3.3 \pm 0.8$ & $3.2 \pm 1.2$ & - \\
\hline & 1960-2099 & $7.2 \pm 0.4$ & - & - & - & - \\
\hline \multirow[t]{3}{*}{ Annual } & 1960-99 & $6.5 \pm 2.9$ & $1.2 \pm 2.2$ & $7.7 \pm 3.1$ & $7.7 \pm 3.8$ & $5.4 \pm 3.0$ \\
\hline & 2000-99 & $6.3 \pm 0.7$ & $0.4 \pm 0.7$ & $5.5 \pm 0.7$ & $6.6 \pm 1.0$ & - \\
\hline & 1960-2099 & $5.9 \pm 0.4$ & - & - & - & - \\
\hline
\end{tabular}

the height region above (Haynes et al. 1991). To illustrate this, Figs. $8 \mathrm{~b}$ and $9 \mathrm{~b}$ show wave drag anomaly time series at $50 \mathrm{hPa}$, a level that is slightly higher than shown for $\bar{w}^{*}$ and representative of the wave drag in the region above (see Fig. 11). As with $\bar{w}^{*}$, in SON the wave drag decreases from 1960 to 2000 and increases thereafter, while in DJF the reverse occurs. Since the decreasing future downwelling in DJF (Fig. 9a) leads to decreasing adiabatic warming, the warming in this time period (Fig. 2b) can only be explained by an overcompensation by increasing solar heating as ozone levels recover.

As will be demonstrated in the next three figures, the wave drag changes are closely related to mean wind changes. Figure 10 (middle panels) shows the seasonal cycle of the linear trends in zonal mean zonal winds averaged from $40^{\circ}$ to $80^{\circ} \mathrm{S}$ for the ODS simulation. Note that different contour intervals are used for the past and future. For reference, the climatological mean winds computed from the first $10 \mathrm{yr}$ of the three simulations combined are shown in Fig. 10a. The past (Fig. 10c) is characterized by large positive trends, which peak at $\sim 5 \mathrm{~m} \mathrm{~s}^{-1}$ decade $^{-1}$ at $10 \mathrm{hPa}$ in November. The trends reverse in the future (Fig. 10d) and are much weaker. The zonal wind trends are primarily a consequence of the thermal wind response to the decrease in solar heating during the period of ozone depletion and the increase during the period of recovery.

The corresponding resolved wave drag trends are shown in Fig. 11. (As in Fig. 10, different contour intervals are used.) Prior to early November there is a positive trend in the past (Fig. 11c), indicating weakening wave drag. This is followed by a strengthening in summer. As ozone (a) SH downward mass flux (ODS SON)

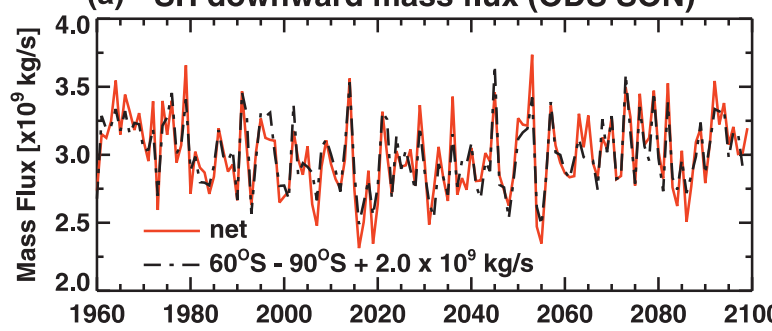

(c) SH downward mass flux (ODS DJF)

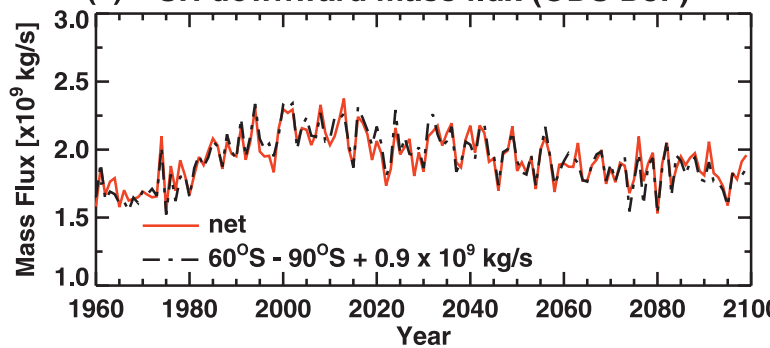

(b) SH downward mass flux (GHG SON)

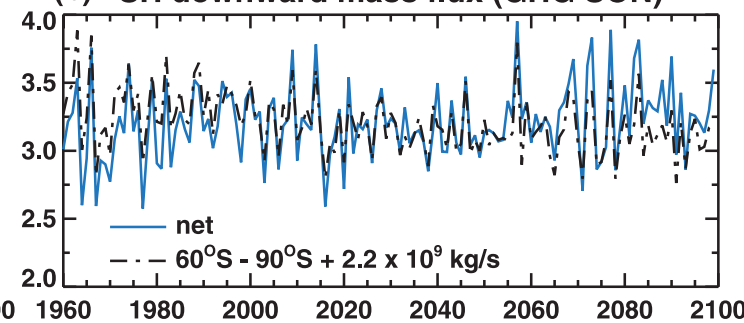

(d) SH downward mass flux (GHG DJF)

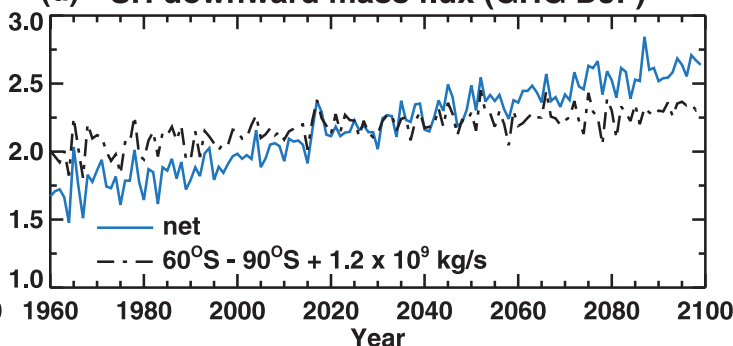

FIG. 7. Time series of SH downward mass flux at $70 \mathrm{hPa}$ for (a),(b) SON and (c),(d) DJF for the (left) ODS and (right) GHG simulations. The colored curves denote the net flux, and the dashed curves the flux poleward of $60^{\circ} \mathrm{S}$. For ease of comparison the dashed curves have been shifted by a constant amount given by the difference in the 140 -yr means of the net and unshifted $60^{\circ}-90^{\circ} \mathrm{S}$ fluxes; the value of the shift is plotted in each panel in units of $\mathrm{kg} \mathrm{s}^{-1}$. Ensemble averages are shown. 


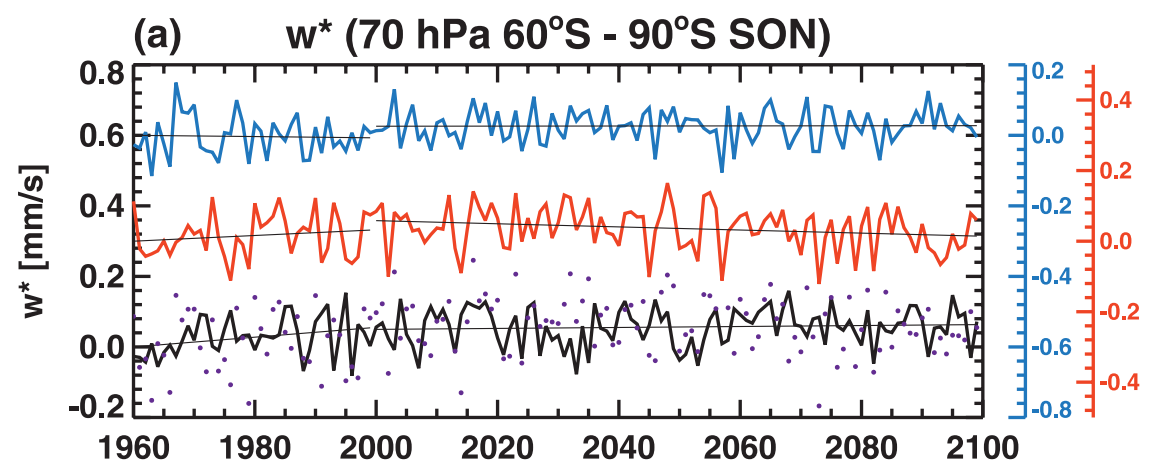

(b) EPFD (50 hPa $40^{\circ} \mathrm{S}-80^{\circ} \mathrm{S}$ SON)

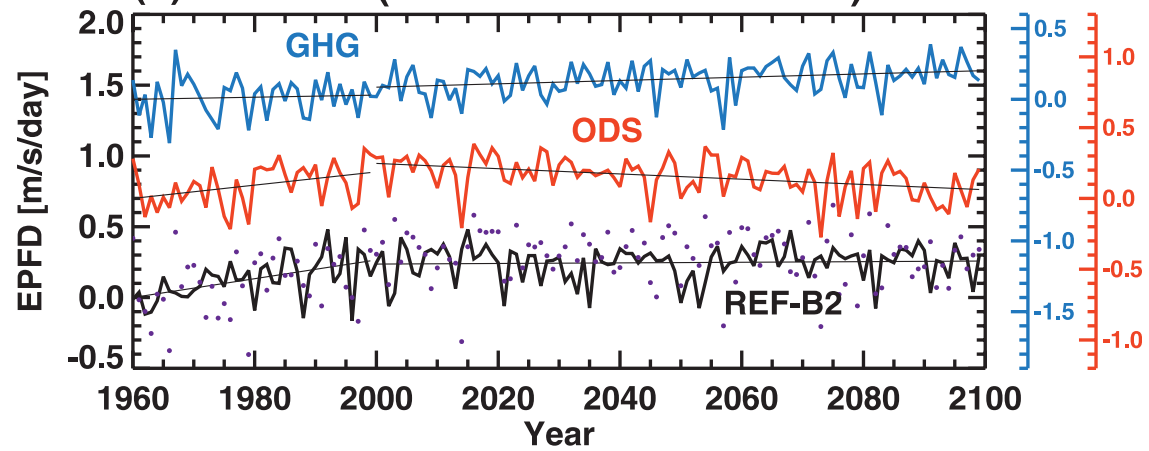

FIG. 8. Time series of (a) residual vertical velocity anomalies $\bar{w}^{*}$ at $70 \mathrm{hPa}$ averaged from $60^{\circ}$ to $90^{\circ} \mathrm{S}$ and (b) EP flux divergence anomalies (EPFD) at $50 \mathrm{hPa}$ averaged from $40^{\circ}$ to $80^{\circ} \mathrm{S}$ for SH spring (SON): GHG (blue), ODS (red), and REF-B2 (black) simulations, and the sum of the GHG and ODS simulations (purple dots). Anomalies are computed with respect to 1960 baselines, which for the REF-B2, GHG, and ODS simulations are, respectively, $-0.30,-0.28$, and $-0.29 \mathrm{~mm} \mathrm{~s}^{-1}$ for $\bar{w}^{*}$ and $-0.55,-0.50$, and $-0.52 \mathrm{~m} \mathrm{~s}^{-1}$ day ${ }^{-1}$ for EPFD. See the Fig. 2 caption for more details.

recovers, the wave drag trends reverse (Fig. 11d). The change in sign of the wave drag trend between spring and summer in the past is a consequence of the delayed breakdown of the $\mathrm{SH}$ vortex caused by ozone depletion. To understand the effects of the winds, Fig. 12 shows time series of the 10-yr mean height of the zero-wind line in December. In the ODS simulation (red curve), the mean height of the zero-wind line increases by over $5 \mathrm{~km}$ between 1960 and the early 2000s, gradually decreasing in the following period. The upward shift during the ozone hole period allows planetary waves to propagate higher into the summer stratosphere, where they dissipate at their critical lines, driving increased downwelling. The zonal wavenumber decomposition of the resolved wave drag presented in MS09 confirmed that it is planetary waves that are responsible.

While the ozone-hole-induced increase in wave drag that drives the increased downwelling in SH summer has been known for some time (Manzini et al. 2003), a decrease in wave drag in spring has, to our knowledge, never been noted. The reason for it is unclear, but may be due to reduced vertical propagation of planetary waves by the strengthening westerlies, which is similar to the mechanism proposed by Plumb (1989) to explain the midwinter minimum in stationary planetary waves in the $\mathrm{SH}$. Another possible explanation could be changes in the resonance properties of the atmosphere, the mechanism proposed by Scott and Haynes (2002) to explain the observed planetary wave structure in the SH. A decrease in the dynamical heating in the Antarctic lower stratosphere in SH spring is also noted in passing in the modeling study of Keeley et al. (2007), but those authors did not investigate changes in wave drag.

\section{2) IMPACTS OF CLIMATE CHANGE}

Turning now to the GHG simulation, climate change causes monotonic changes in the polar cap downwelling and midlatitude wave drag, as seen in Figs. 8 and 9 (blue curves). In SON (Fig. 8), weakening wave drag causes reduced downwelling. This is attributed to reduced upward planetary wave flux in the upper troposphere (MS09). In DJF (Fig. 9), the sign of the trends reverses, with increasing wave drag causing increasing downwelling. 


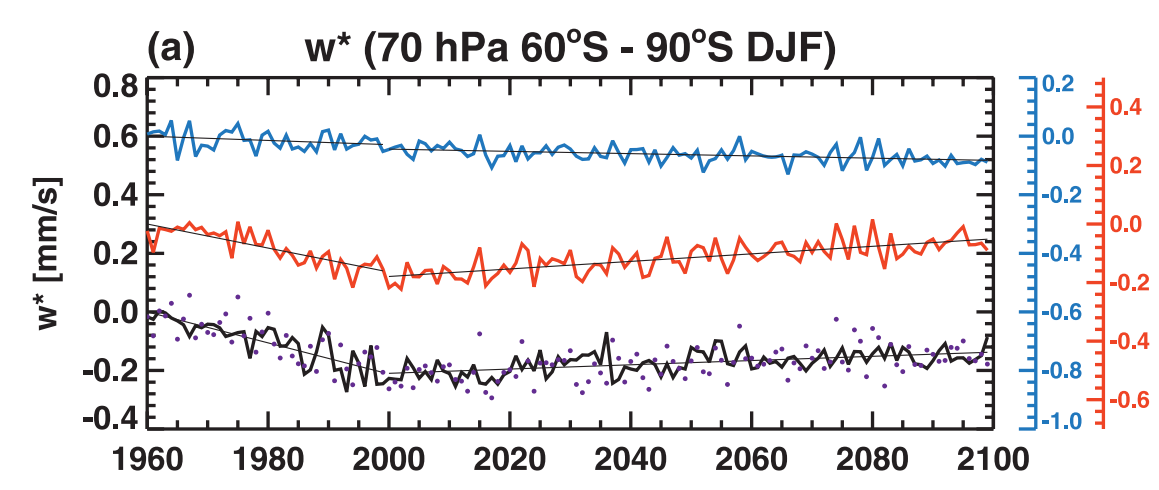

(b) EPFD (50 hPa $40^{\circ} \mathrm{S}-80^{\circ} \mathrm{S}$ DJF)

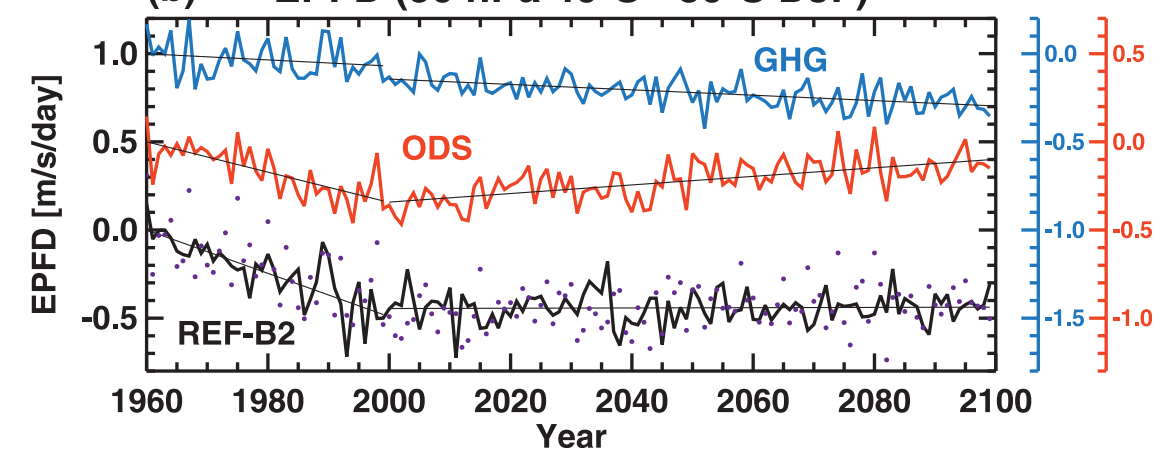

FIG. 9. As in Fig. 8, but for SH summer (DJF). The 1960 baselines for the REF-B2, GHG, and ODS simulations are, respectively, $-0.14,-0.18$, and $-0.16 \mathrm{~mm} \mathrm{~s}^{-1}$ for $\bar{w}^{*}$ and $-0.57,-0.71$, and $-0.64 \mathrm{~m} \mathrm{~s}^{-1} \mathrm{day}^{-1}$ for EPFD.

The cause of the seasonal differences in trends in the GHG simulation can also be understood by considering the monthly changes in the mean wind and resolved wave drag trends (Figs. 10b and 11b). The decrease in wave drag in spring delays the breakdown of the polar vortex, which results in favorable conditions for the vertical propagation of Rossby waves in early summer. This allows planetary waves to propagate slightly higher into the summer stratosphere where they are dissipated, as in the ODS simulation. The steady increase with time of the height of the zero-wind line in December (blue curve in Fig. 12), with easterlies overlaying westerlies, is what allows for this enhanced vertical propagation and dissipation, producing the increased downwelling in the region below. (The changes in the height of the zero-wind line cannot be the result of the stratospheric wave drag changes at that time, as the latter would tend to induce easterlies rather than westerlies.)

The REF-B2 simulation exhibits characteristics of both the ODS and GHG simulations. The polar cap downwelling and midlatitude wave drag computed from the sum of the two sensitivity experiments (dotted curves in Figs. 8 and 9) are in very good agreement with the REF-B2 results. This agreement is further quantified in Table 1.

\section{c. Breakdown of the polar vortex}

The monthly mean zonal wind data presented above show that climate change and ozone depletion both cause a delay in the breakdown of the polar vortex. To better quantify these changes, daily data are employed. Figure 13 shows 20-yr means of the height of the zero-wind line at $60^{\circ} \mathrm{S}$ versus the day of the year for four different time periods. To aid in the comparison, a baseline profile computed from the first $10 \mathrm{yr}$ of the three sets of simulations is plotted in each panel (dotted curve). In the past (Fig. 13a), the profiles all overlay the baseline, indicating that GHG and ODS concentrations have not increased sufficiently to cause a change in the breakdown of the vortex. In the present (Fig. 13b), the zero-wind line for the ODS simulation (red curve) has shifted by 10-15 days with respect to the baseline, while that for the GHG simulation (blue curve) is largely unchanged. Cooling of the polar vortex due to enhanced ozone depletion causes this shift; climate change has still not had enough time to make its mark. By the middle of this century (Fig. 13c), all three simulations are nearly the same but are shifted $\sim 10$ days with respect to the baseline. This occurs because ozone recovery is restoring the vortex to its 1960 value, while climate change is delaying its breakdown. 


\section{Zonal mean zonal wind $\left(40^{\circ} \mathrm{S}-80^{\circ} \mathrm{S}\right)$}
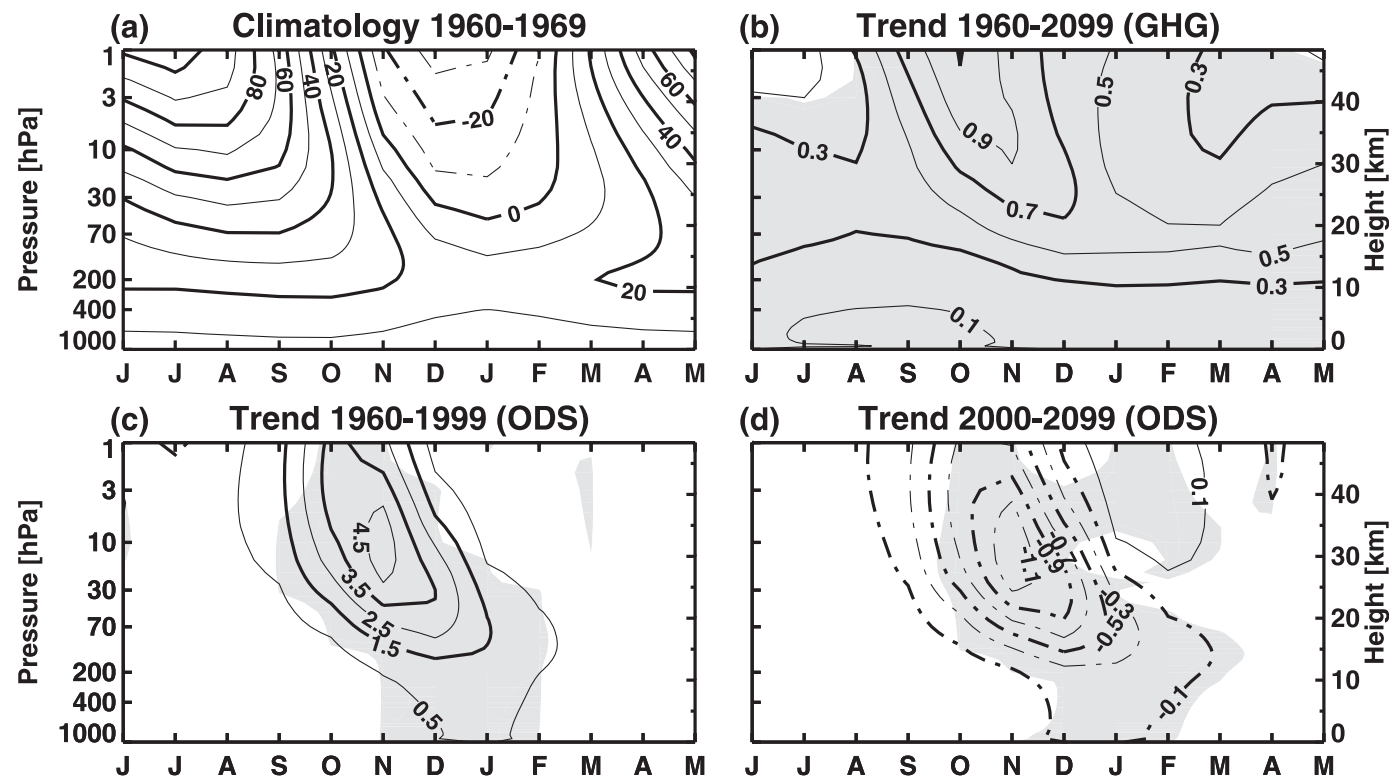

(e) Trend 1960-1999 (REF-B2)
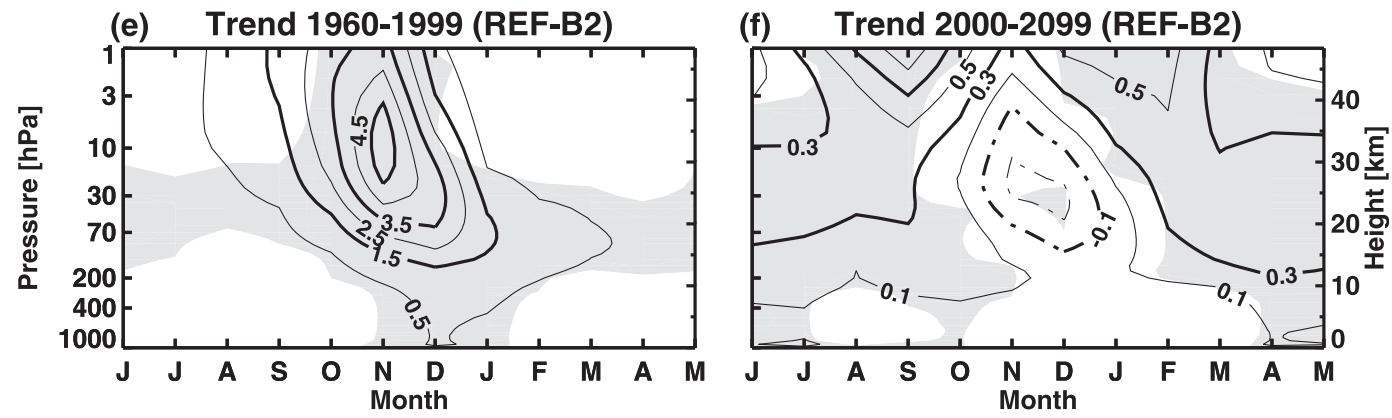

FIG. 10. Monthly time series of zonal and monthly mean zonal wind averaged from $40^{\circ}$ to $80^{\circ} \mathrm{S}$. (a) Past climatology (1960-69) for the three sets of simulations combined. Linear trends from (b) 1960-2099 for the GHG simulations, (c) 1960-99 and (d) 2000-99 for the ODS simulations, and (e) 1960-99 and (f) 2000-99 for the REF-B2 simulations. Gray shading denotes confidence levels $\geq 95 \%$ that the trends are different from zero. Contour intervals are $10 \mathrm{~m} \mathrm{~s}^{-1}$ in (a), $1 \mathrm{~m} \mathrm{~s}^{-1}$ decade $^{-1}$ in (c) and (e), and $0.2 \mathrm{~m} \mathrm{~s}^{-1}$ decade $^{-1}$ in (b),(d), and (f). Ensemble averages are shown.

At the end of this century (Fig. 13d), ozone has sufficiently recovered so that the ODS simulation has nearly returned to its initial value, with climate change now controlling the position of the zero-wind line, which is shifted 10-15 days from the baseline. In all cases the shifts in the zero-wind line extend throughout the entire stratosphere, decreasing slightly with increasing height. It is noteworthy that Fig. 13c shows an exception to the additivity seen in other diagnostics since the perturbations from the baseline profile for the GHG and ODS simulations do not add up to the REF-B2 perturbation for this time period. In fact, nonadditivity is also seen over the same time period in the height of the zero-wind line in December (Fig. 12). The additivity of the zonal wind changes discussed previously refers to trends over the two multidecadal time periods. It does not necessarily preclude some nonadditivity in midcentury when the effects of GHGs and ODSs are of comparable magnitude (Fig. 1).

Time series of final warming dates at $50 \mathrm{hPa}$ are shown in Fig. 14 for each member of the three sets of ensembles. Final warmings are computed using the same method as that of Black and McDaniel (2007): zonal mean zonal winds at $60^{\circ} \mathrm{S}$ are first smoothed using a 5-day low-pass filter and warmings identified when the smoothed winds first fall below $10 \mathrm{~m} \mathrm{~s}^{-1}$ and remain below that threshold until the following winter. The GHG simulation (Fig. 14a) shows a gradual monotonic increase in the final warming date, with a trend of 1.3 days decade ${ }^{-1}$ over the entire simulation (not shown). In the ODS simulation (Fig. 14b) a strong positive trend of 9.1 days decade ${ }^{-1}$ occurs prior to 2000 , followed by a weak negative trend. In the REF-B2 
EP flux divergence $\left(40^{\circ} \mathrm{S}-8^{\circ} \mathrm{S}\right)$
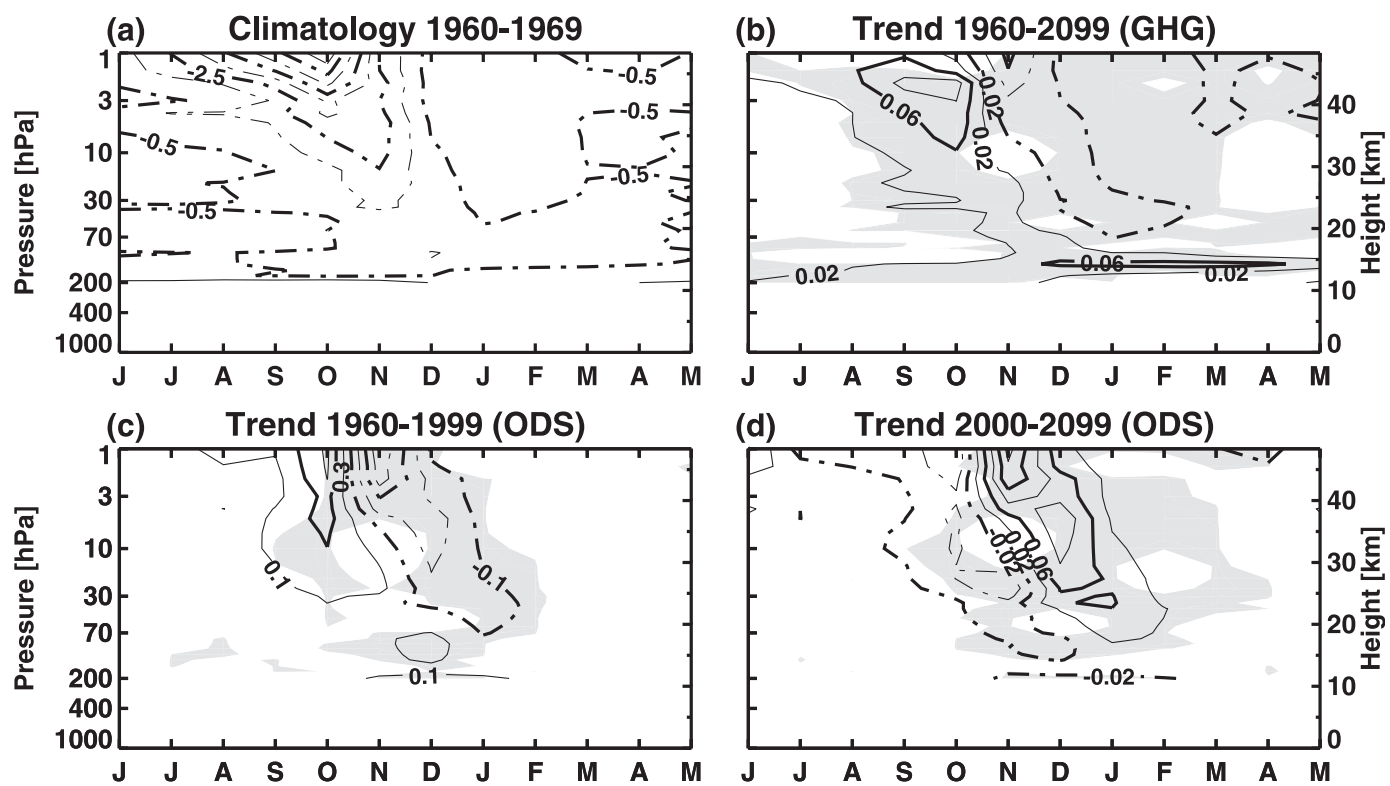

(e) Trend 1960-1999 (REF-B2)

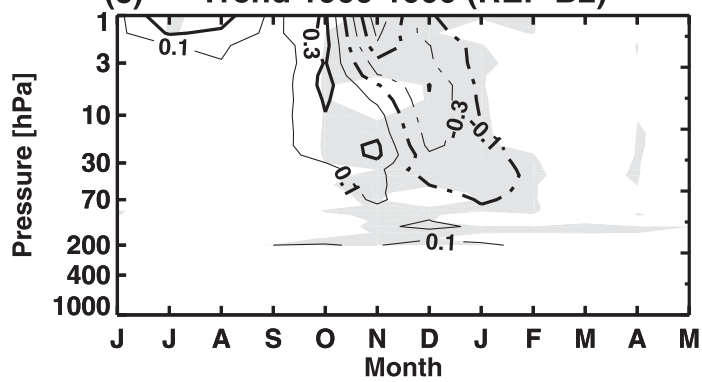

(f)

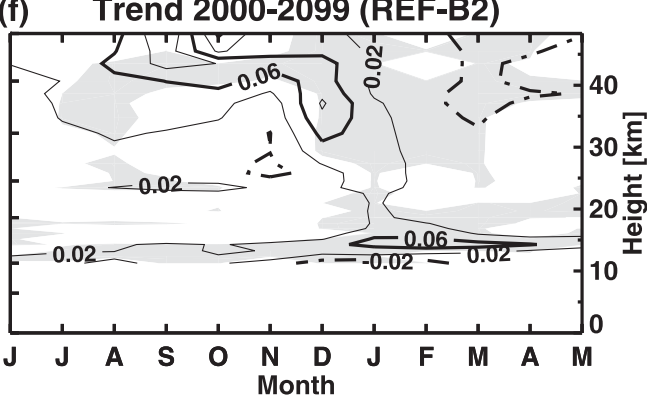

FIG. 11. As in Fig. 10, but for the Eliassen-Palm flux divergence. Contour intervals are $1 \mathrm{~m} \mathrm{~s}^{-1} \mathrm{day}^{-1}$ in (a), $0.2 \mathrm{~m} \mathrm{~s}^{-1}$ day $^{-1}$ decade $^{-1}$ in (c) and (e), and $0.04 \mathrm{~m} \mathrm{~s}^{-1}$ day $^{-1}$ decade $^{-1}$ in (b),(d), and (f). Positive trends indicate decreasing wave drag. Contours not plotted below $200 \mathrm{hPa}$.

simulation (Fig. 14c), the trends reflect the combined effects of climate change and ozone depletion and recovery, and are additive within statistical uncertainty. As indicated by the plotted uncertainties, all trends are significantly different from zero at the $95 \%$ level.

The Antarctic vortex in most CCMs (including CMAM) breaks down about 2-3 weeks too late (Eyring et al. 2006; SPARC CCMVal 2010, chapter 4). This bias is a longstanding issue whose cause is still not understood. Nevertheless, in spite of this bias the REF-B2 trend in the final warming date shown in Fig. 14 closely matches (within the uncertainties) the observed trend of 10.9 days decade ${ }^{-1}$ (Black and McDaniel 2007).

\section{Summary and conclusions}

The Canadian Middle Atmosphere Model is used to assess the separate effects of climate change and ozone depletion (recovery) on the dynamics of the SH stratosphere. CMAM is unique among models participating in CCMVal-2 in that it is coupled to an ocean general circulation model. This enables a two-way feedback between the ocean and atmosphere, a process that is missing in all other models that use prescribed SSTs. Coupling to an ocean model yields not only seamless simulations from past to future, but also SSTs that respond in a selfconsistent manner to the imposed anthropogenic forcings.

Three sets of simulations extending from 1960 to 2099 are analyzed: 1) the GHG simulations in which ODSs are held fixed at their 1960 levels and GHGs vary in time, 2) the ODS simulations in which GHGs are held fixed at their 1960 levels and ODSs vary in time, and 3) the REFB2 simulations in which both GHGs and ODSs vary in time. Since each set of simulations comprises an ensemble of three members, a good signal-to-noise ratio is obtained, enabling the physical responses to be 


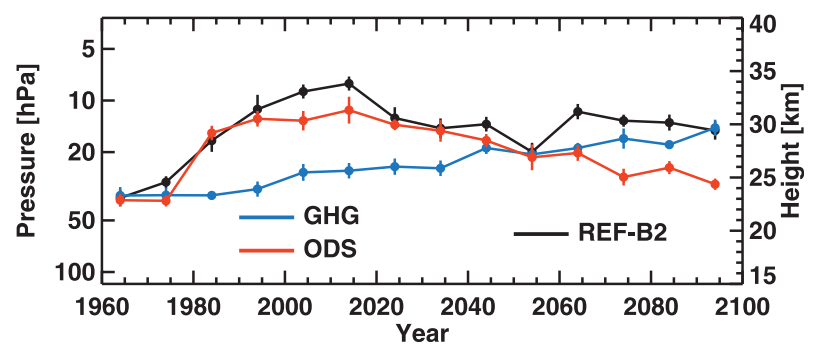

FIG. 12. Time series of the height of the zero-wind line computed from December monthly and zonal mean zonal wind profiles averaged from $50^{\circ}$ to $90^{\circ} \mathrm{S}$ : GHG (blue), ODS (red), and REF-B2 (black) simulations. The 10-yr means centered at the positions of the filled circles are shown. The error bars are the standard errors of the 10-yr means. Ensemble averages are shown.

identified with a higher degree of confidence. The two sensitivity experiments (i.e., the first and second sets) enable the separate effects of climate change and ozone depletion-recovery to be studied; the three together enable the question of additivity to be addressed.

In almost all of the physical quantities analyzed (zonal mean temperature and zonal wind, mass flux, residual vertical velocity, resolved wave drag, and final warming date), the stratospheric response to GHG and ODS forcings is found to be additive within statistical uncertainty. This was determined by comparing the long-term trends computed from the sum of the two sensitivity experiments to the trends from the simulation using the combined time-varying GHG and ODS forcings. Additivity does not mean, however, that there is no nonlinearity in the response, only that, if there is, the signal is too weak to be detected. The only diagnostics that exhibited nonadditivity were the delay in the transition to polar easterlies throughout most of the stratosphere in the middle of the twenty-first century and the corresponding height of the zero-wind line in December, which are of course related. This time period is when the ODS and GHG effects on these diagnostics are of comparable magnitude, which is when one might expect the strongest nonadditivity.

An examination of the ODS simulation reveals that ozone depletion and recovery has an impact on the lower stratosphere net upward mass flux in SON and DJF. Surprisingly, the trends in the two seasons are of

Transition to easterlies at $60^{\circ} \mathrm{S}$
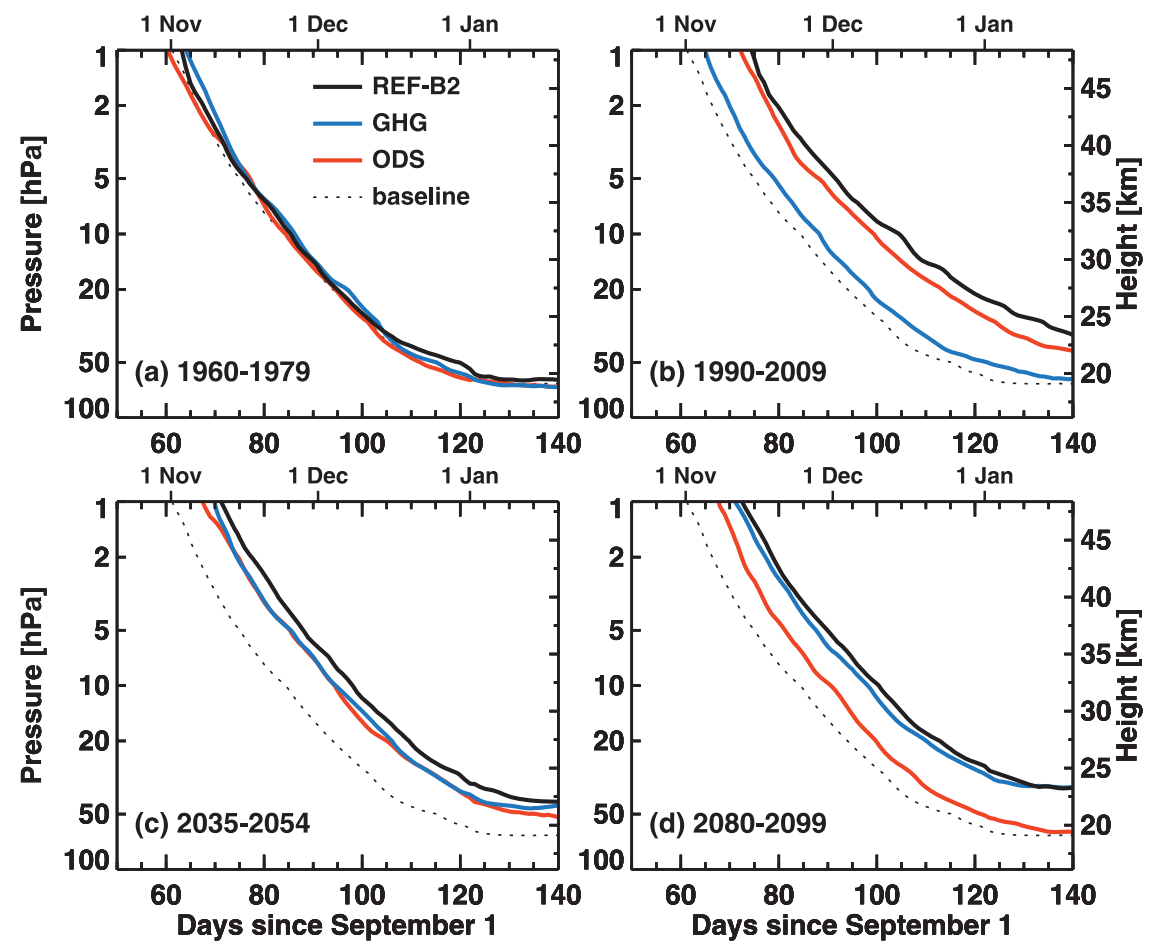

FIG. 13. Height of the zero-wind line at $60^{\circ} \mathrm{S}$ vs day of the year computed from daily zonal mean zonal winds from (a) 1960-79, (b) 1990-2009, (c) 2035-54, and (d) 2080-99: GHG (blue), ODS (red), and REF-B2 (black) simulations. The dotted line is a baseline given by the average of the first $10 \mathrm{yr}$ (1960-69) of the three sets of simulations, and is replotted for reference in each panel. Ensemble averages are shown. 


\section{Final warming dates at $60^{\circ} \mathrm{S} \& 50 \mathrm{hPa}$}
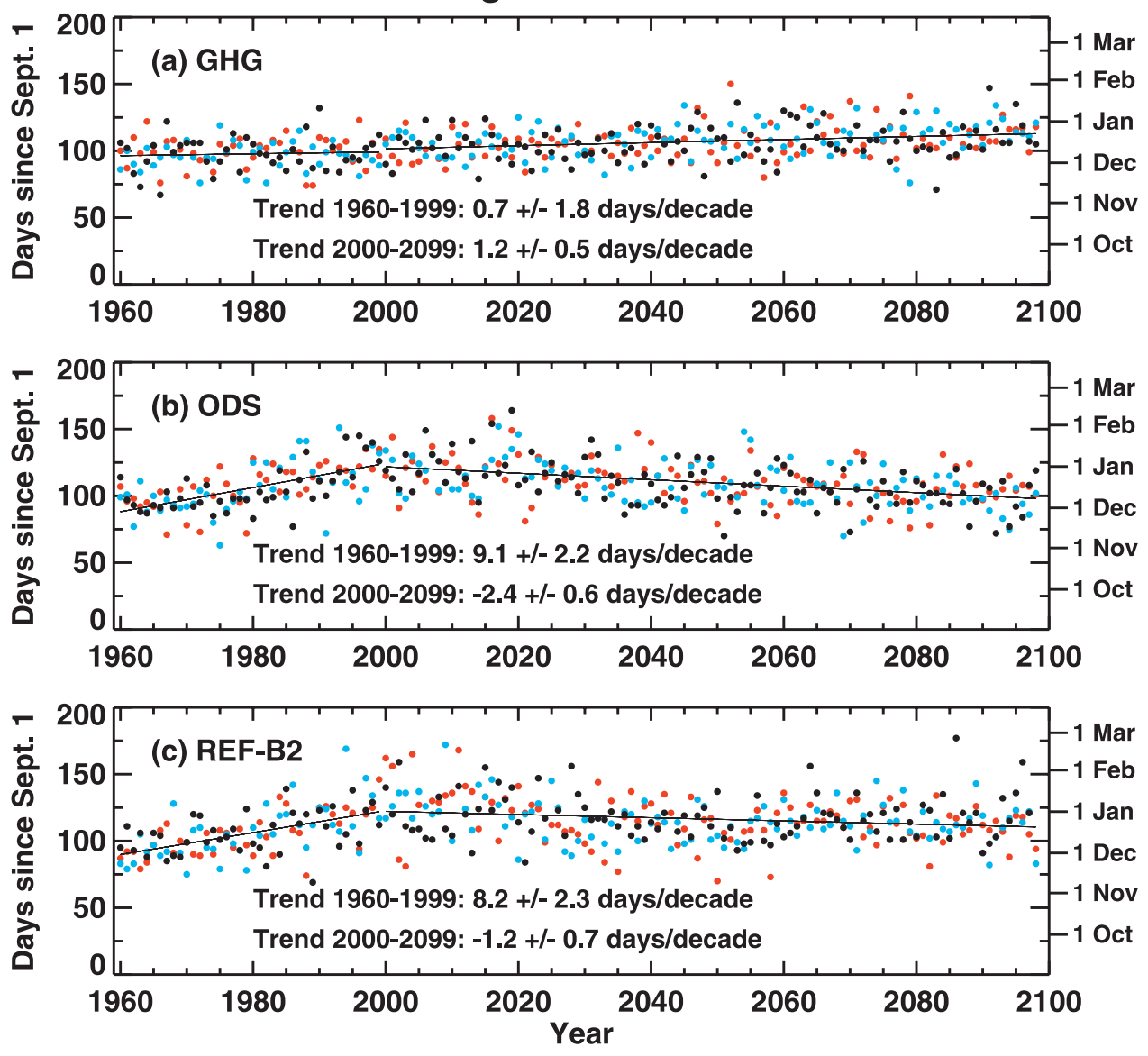

FIG. 14. Time series of final warming dates at $50 \mathrm{hPa}$ computed from daily zonal mean zonal winds at $60^{\circ} \mathrm{S}$ : (a) GHG, (b) ODS, and (c) REF-B2 simulations. Colors denote the different ensemble members for each set of simulations. The straight lines are linear fits computed from 1960-99 and 2000-99; the corresponding slopes and their uncertainties (95\% confidence levels) are plotted in each panel.

opposite sign: in SON negative trends in the past (196099) are followed by positive trends in the future (200099), while in DJF positive trends in the past are followed by negative trends in the future. An examination of the net downward mass flux indicates that the changes are coming from the SH. In the other two seasons, and in the annual mean, the mass flux trends in the ODS simulation are much weaker, in the latter case because of the partial cancelation in the trends for SON and DJF. Climate change (GHG simulation), on the other hand, is found to be the most important driver of the mass flux trends in the REF-B2 simulation, reconfirming previous results (e.g., Butchart et al. 2006) that climate change is responsible for the acceleration of the Brewer-Dobson circulation in model simulations.

The oppositely signed net upward mass flux trends in SON and DJF in the ODS simulation are attributed to seasonal differences in SH polar cap average downwelling trends in the lower stratosphere. The changes in downwelling are in turn related to changes in resolved wave drag in the middle stratosphere at mid- to high southern latitudes. In the past, wave drag exhibits a positive trend (weakening) in spring that peaks in October, reversing sign in summer. The negative trend (strengthening) in summer is a consequence of the delayed breakdown of the SH vortex brought about by the increased cooling that results from the reduced ozone concentrations. This raises the height of the zero-wind line, enabling Rossby waves to propagate higher into the summer stratosphere where they dissipate and drive the increased downwelling. This mechanism has been discussed in a number of model studies (Manzini et al. 2003; Li et al. 2008; MS09) and been inferred from ozone measurements (Stolarski et al. 2006). However, the springtime decrease in wave drag during the ozone hole period and the corresponding increase during the ozone recovery period, to our knowledge, have not previously been noted. Whether this occurs in other CCMs or in the 
observations is unknown at present. While its cause is difficult to identify with certainty, it is most likely related to changes in the zonal mean background state, as opposed to changes in tropospheric forcing: the increase in the westerlies in springtime may reduce the vertical propagation of planetary waves (Plumb 1989) or change the resonance properties of the mean flow (Scott and Haynes 2002).

In the GHG simulation, a steady decrease with time in polar cap downwelling occurs in $\mathrm{SH}$ spring. This is attributed to the reduction in the upward flux of planetary wave activity from the troposphere that is due to climate change (MS09). In summer there is a steady increase in downwelling in response to increasing wave drag that results from the climate-change-induced delay in the breakdown of the SH vortex and the associated steady increase with time in the height of the zero-wind line in summer, the same mechanism that operates in the ODS simulation in summer during the period of increasing ozone depletion.

Daily zonal mean zonal winds at $60^{\circ} \mathrm{S}$ are used to quantify the changes in the date of the breakdown of the SH vortex. At the end of the twentieth century the vortex breaks down 10-15 days later than in the early 1960s as a result of ozone depletion. The trend in final warming dates at $50 \mathrm{hPa}$ that is implied from this is in good agreement (within statistical uncertainty) of the observed 10.9 days decade ${ }^{-1}$ trend (Black and McDaniel 2007). By the middle of this century, the breakdown dates for the two sensitivity experiments are about the same (with climate change delaying breakdown and ozone recovery advancing it), but both are shifted about 10 days later with respect to the early 1960s. By the end of this century, climate change explains almost all of the 10-15-day delay in breakdown. Trends in final warming dates, which are statistically significant at the $95 \%$ level, are found to be additive within the noise level.

Acknowledgments. The authors thank John Austin, four other reviewers, and Nathan Gillett for their constructive comments on the manuscript. This work was supported by the Canadian Foundation for Climate and Atmospheric Sciences through the C-SPARC project.

\section{REFERENCES}

Andrews, D. G., J. R. Holton, and C. B. Leovy, 1987: Middle Atmosphere Dynamics. Academic Press, 489 pp.

Arora, V. K., and Coauthors, 2009: The effect of terrestrial photosynthesis down-regulation on the 20th century carbon budget simulated with the CCCma Earth System Model. J. Climate, 22, 6066-6088.

Black, R. X., and B. A. McDaniel, 2007: Interannual variability in the Southern Hemisphere circulation organized by stratospheric final warming events. J. Atmos. Sci., 64, 2968-2974.
Butchart, N., and Coauthors, 2006: Simulations of anthropogenic change in the strength of the Brewer-Dobson circulation. Climate Dyn., 27, 727-741, doi:10.1007/s00382-006-0162-4.

de Grandpré, J., S. R. Beagley, V. I. Fomichev, E. Griffioen, J. C. McConnell, A. S. Medvedev, and T. G. Shepherd, 2000: Ozone climatology using interactive chemistry: Results from the Canadian middle atmosphere model. J. Geophys. Res., 105, 26 475-26 491.

Eyring, V., and Coauthors, 2006: Assessment of temperature, trace species, and ozone in chemistry-climate model simulations of the recent past. J. Geophys. Res., 111, D22308, doi:10.1029/ 2006JD007327.

- and Coauthors, 2008: Overview of the new CCMVal reference and sensitivity simulations in support of upcoming ozone and climate assessments and the planned SPARC CCMVal report. SPARC Newsletter, No. 30, Stratospheric Processes and their Role in Climate, World Climate Research Programme, 20-26.

Garcia, R. R., and W. J. Randel, 2008: Acceleration of the BrewerDobson circulation due to increases in greenhouse gases. J. Atmos. Sci., 65, 2731-2739.

Gent, P. R., and Coauthors, 1998: The NCAR Climate System Model global ocean component. J. Climate, 11, 1287-1306.

Gillett, N. P., and D. W. J. Thompson, 2003: Simulation of recent Southern Hemisphere climate change. Science, 302, 273-275.

Haynes, P. H., C. J. Marks, M. E. McIntyre, T. G. Shepherd, and K. P. Shine, 1991: On the "downward control" of extratropical diabatic circulations by eddy-induced mean zonal forces. J. Atmos. Sci., 48, 651-678.

Holton, J. R., 1990: On the global exchange of mass between the stratosphere and troposphere. J. Atmos. Sci., 47, 392-395.

Houghton, J. T., Y. Ding, D. J. Griggs, M. Noguer, P. J. van der Linden, X. Dai, K. Maskell, and C. A. Johnson, Eds., 2001: Climate Change 2001: The Scientific Basis. Cambridge University Press, 881 pp.

Jonsson, A. I., V. I. Fomichev, and T. G. Shepherd, 2009: The effect of nonlinearity in $\mathrm{CO}_{2}$ heating rates on the attribution of stratospheric ozone and temperature changes. Atmos. Chem. Phys., 9, 8447-8452.

Keeley, S. P. E., N. P. Gillett, D. W. J. Thompson, S. Solomon, and P. M. Forster, 2007: Is Antarctic climate most sensitive to ozone depletion in the middle or lower stratosphere? Geophys. Res. Lett., 34, L22812, doi:10.1029/2007GL031238.

Langematz, U., M. Kunze, K. Krüger, K. Labitzke, and G. L. Roff, 2003: Thermal and dynamical changes of the stratosphere since 1979 and their link to ozone and $\mathrm{CO}_{2}$ changes. J. Geophys. Res., 108, 4027, doi:10.1029/2002JD002069.

Li, F., J. Austin, and J. Wilson, 2008: The strength of the BrewerDobson circulation in a changing climate: Coupled chemistryclimate model simulations. J. Climate, 21, 40-57.

Manzini, E., B. Steil, C. Brühl, M. A. Giorgetta, and K. Krüger, 2003: A new interactive chemistry-climate model: 2 . Sensitivity of the middle atmosphere to ozone depletion and increase in greenhouse gases and implications for recent stratospheric cooling. J. Geophys. Res., 108, 4429, doi:10.1029/2002JD002977.

McLandress, C., and T. G. Shepherd, 2009: Simulated anthropogenic changes in the Brewer-Dobson circulation, including its extension to high latitudes. J. Climate, 22, 15161540.

Oman, L., D. W. Waugh, S. Pawson, R. S. Stolarski, and P. A. Newman, 2009: On the influence of anthropogenic forcings on changes in the stratospheric mean age. J. Geophys. Res., 114, D03105, doi:10.1029/2008JD010378. 
Perlwitz, J., S. Pawson, R. L. Fogt, J. E. Nielsen, and W. D. Neff, 2008: Impact of stratospheric ozone hole recovery on Antarctic climate. Geophys. Res. Lett., 35, L08714, doi:10.1029/ 2008GL033317.

Pitts, M. C., L. W. Thomason, L. R. Poole, and D. M. Winker, 2007: Characterization of polar stratospheric clouds with spaceborne lidar: CALIPSO and the 2006 Antarctic season. Atmos. Chem. Phys., 7, 5207-5228.

Plumb, R. A., 1989: On the seasonal cycle of stratospheric planetary waves. Pure Appl. Geophys., 130, 233-242.

Plummer, D. A., J. F. Scinocca, T. G. Shepherd, M. C. Reader, and A. I. Jonsson, 2010: Contributions to stratospheric change from ozone depleting substances and greenhouse gases. Atmos. Chem. Phys. Discuss., 10, 9647-9694, doi:10.5194/acpd-10-96472010.

Randel, W. J., and Coauthors, 2009: An update of observed stratospheric temperature trends. J. Geophys. Res., 114, D02107, doi:10.1029/2008JD010421.

Santee, M. L., and Coauthors, 2008: A study of stratospheric chlorine partitioning based on new satellite measurements and modeling. J. Geophys. Res., 113, D12307, doi:10.1029/ 2007JD009057.

Scinocca, J. F., N. A. McFarlane, M. Lazare, J. Li, and D. Plummer, 2008: The CCCma third generation AGCM and its extension into the middle atmosphere. Atmos. Chem. Phys., 8, 70557074.

, M. C. Reader, D. A. Plummer, M. Sigmond, P. J. Kushner, T. G. Shepherd, and A. R. Ravishankara, 2009: Impact of sudden Arctic sea-ice loss on stratospheric polar ozone recovery. Geophys. Res. Lett., 36, L24701, doi:10.1029/2009GL041239.

Scott, R. K., and P. H. Haynes, 2002: The seasonal cycle of planetary waves in the winter stratosphere. J. Atmos. Sci., 59, 803-822.
Shepherd, T. G., 2008: Dynamics, stratospheric ozone, and climate change. Atmos.-Ocean, 46, 371-392.

— ozone and temperature changes to changes in ozone-depleting substances and well-mixed greenhouse gases. Atmos. Chem. Phys., 8, 1435-1444.

Shine, K. P., and Coauthors, 2003: A comparison of model-simulated trends in stratospheric temperatures. Quart. J. Roy. Meteor. Soc., 129, 1565-1588.

SPARC CCMVal, 2010: SPARC report on the evaluation of chemistry-climate models. V. Eyring, T. G. Shepherd, and D. W. Waugh, Eds., SPARC Rep. 5, WCRP-132, WMO/TD1526. [Available online at http://www.atmosp.physics.utoronto. ca/SPARC.]

Stolarski, R. S., A. R. Douglass, M. Gupta, P. A. Newman, S. Pawson, M. R. Schoeberl, and J. E. Nielsen, 2006: An ozone increase in the Antarctic summer stratosphere: A dynamical response to the ozone hole. Geophys. Res. Lett., 33, L21805, doi:10.1029/2006GL026820.

,-- , P. A. Newman, S. Pawson, and M. R. Schoeberl, 2010: Relative contribution of greenhouse gases and ozone-depleting substances to temperature trends in the stratosphere: A chemistry-climate model study. J. Climate, 23, 28-42.

Waugh, D. W., W. J. Randel, S. Pawson, P. A. Newman, and E. R. Nash, 1999: Persistence of the lower stratospheric polar vortices. J. Geophys. Res., 104, 27 191-27 202.

, L. Oman, S. R. Kawa, R. S. Stolarski, S. Pawson, A. R. Douglass, P. A. Newman, and J. E. Nielsen, 2009: Impacts of climate change on stratospheric ozone recovery. Geophys. Res. Lett., 36, L03805, doi:10.1029/2008GL036223.

WMO, 2007: Scientific assessment of ozone depletion: 2006. Global Ozone Research Monitoring Project Rep. 50, WMO, Geneva, Switzerland. 